\title{
Rotational periods of solar-mass young stars in Orion $\star, \star \star$
}

\author{
E. Marilli ${ }^{1}$, A. Frasca ${ }^{1}$, E. Covino ${ }^{2}$, J. M. Alcalá ${ }^{2}$, S. Catalano ${ }^{1}$, M. Fernández ${ }^{3}$, \\ A. Arellano Ferro ${ }^{4}$, E. Rubio-Herrera ${ }^{4}$, and L. Spezzi ${ }^{1}$ \\ 1 INAF, Osservatorio Astrofisico di Catania, via S. Sofia, 78, 95123 Catania, Italy \\ e-mail: emarilli@oact.inaf.it \\ 2 INAF, Osservatorio Astronomico di Capodimonte, via Moiariello 16, 80131 Napoli, Italy \\ 3 Instituto de Astrofísica de Andalucía, CSIC, Camino Bajo de Huétor 50, 18008 Granada, Spain \\ ${ }^{4}$ Instituto de Astronomía, Universidad Nacional Autónoma de México, Apdo. Postal 70-264, D.F. CP 04510, México
}

Received 27 September 2006 / Accepted 30 November 2006

\section{ABSTRACT}

\begin{abstract}
Context. The evolution of the angular momentum in young low-mass stars is still a debated issue. The stars presented here were discovered as X-ray sources in the ROSAT All-Sky Survey (RASS) of the Orion complex and subsequently optically identified thanks to both low and high resolution spectroscopy.

Aims. The determination of the rotational periods in young low-mass stars of different age is fundamental for the understanding of the angular momentum evolution.

Methods. We performed a photometric monitoring program on a sample of 40 solar-mass young stars in the Orion star-forming region, almost all previously identified as weak T Tauri stars (WTTS) candidates. Photometric $B$ and $V$ data were collected from 1999 to 2006 at Catania Astrophysical Observatory (OAC). Data were also acquired in December 1998 at Calar Alto Observatory (CA) and in 1999, 2000, and 2003 at San Pedro Martir (SPM). From the observed rotational modulation, induced by starspots, we derived the rotation periods, using both the Lomb-Scargle periodogram and the CLEAN deconvolution algorithms.

Results. In total, we were able to determine the rotation periods for 39 stars, spanning from about 0.5 to 13 days, showing a rather flat distribution with a peak around 1-2 days. Though some of these stars were found to be spectroscopic binaries, only the systems with shorter orbital periods and circular orbits turned out to be synchronized. In the other cases, the rotational period is shorter than the period of pseudo-synchronization at periastron.

Conclusions. The new data provide further evidence for the spin up of solar-mass stars predicted by models of angular momentum evolution of pre-main sequence (PMS) stars.
\end{abstract}

Key words. stars: pre-main sequence - X-ray: stars - stars: activity - techniques: photometric - ISM: individual objects: Orion

\section{Introduction}

The knowledge of the rotation period of low-mass stars at different evolutionary stages is fundamental for studying their angular momentum evolution both from the observational and theoretical point of view. Measurements of low-amplitude periodic light variations, ascribed to the modulation induced by uneven starspot distributions on the stellar photospheres, are a direct way to obtain this physical parameter. Recently, strong efforts have been devoted to the determination of the rotation rate among pre-main sequence (PMS) stars (Herbst \& Mundt 2005; Rebull et al. 2004). Indeed, it is very important to search for periods in the age range between the T Tauri stars (TTS) (age $\approx 1-5 \mathrm{Myr}$ ) and the youngest cluster dwarfs (age $\approx$ $50 \mathrm{Myr}$ ). This age gap might be filled by the oldest weak-T Tauri stars (WTTS) and post-TTS, that have lost the largest part of their circumstellar envelopes and are approaching the ZAMS on their radiative tracks. X-ray observations with the ROSAT satellite, mainly based on the ROSAT All-Sky-Survey (RASS), revealed strong X-ray emitters in star-forming regions (SFRs) and

\footnotetext{
* Based on observations collected at the Catania Astrophysical Observatory (Italy), at the Estación de Observación de Calar Alto (Spain), and San Pedro Martir Observatory (México).

$\star \star$ Appendix A is only available in electronic form at http://www . aanda.org
}

in their surroundings. Recent photometric and spectroscopic investigations have confirmed that many of these objects are bonafide WTTS (Alcalá et al. 2000a; Covino et al. 2001; Frasca et al. 2003). In this work we report the results of a photometric monitoring campaign on 40 optical counterparts of X-ray sources in the Orion complex previously identified as WTTS candidates, both singles and in binary systems. Relevant information on these stars is reported in Table 1, where, for nearly all objects, spectral types come from Alcalá et al. (2000a) and the $V$ magnitude at maximum brightness and $B-V$ color index are derived in the present work. The spectral type of 1RXS J035637.8-132719 has been determined in this work based on three unpublished FEROS spectra.

\section{Observations and data reduction}

The largest part of the photometric observations was performed, in the Johnson $B$ and $V$ bands, at the M.G. Fracastoro station (Serra La Nave, Mt. Etna, $1750 \mathrm{~m}$ a.s.l.) of the Catania Astrophysical Observatory (OAC), with the $0.91 \mathrm{~m}$ Cassegrain telescope, from 1999 to 2006. A single-head photon-counting photometer equipped with an EMI 9893 QA/350 photomultiplier, cooled at $-15^{\circ} \mathrm{C}$, was employed. The data were reduced to the standard Johnson system using local zero-point stars and standard stars selected in some Landolt (1992) areas. The 
Table 1. Targets in our sample.

\begin{tabular}{|c|c|c|c|c|c|c|}
\hline $1 \mathrm{RXS} \mathrm{J/RX} \mathrm{J}$ & Name & Sp. Type ${ }^{a}$ & $V(\mathrm{mag})^{b}$ & $B-V^{b}$ & $\operatorname{Rem}^{a}$ & Obs. \\
\hline $035030.9-135527$ & BD- $14^{\circ} 758$ & $\mathrm{~K} 0-1 / \mathrm{K} 1-2^{d}$ & 10.58 & 0.80 & $\mathrm{SB} 2^{d}$ & OAC(Jan.-Feb. 99)/CA \\
\hline $035637.8-132719$ & & $\mathrm{~K} 1 \mathrm{~V}^{b}$ & 11.76 & 0.97 & Single & OAC(Jan.-Feb. 99)/CA \\
\hline $044059.2-084005$ & BD- $08^{\circ} 913$ & $\mathrm{G} 3 / \mathrm{G}^{d}$ & 10.06 & 0.64 & $\mathrm{SB} 2^{d}$ & OAC(Nov. 99-Feb. 00)/CA \\
\hline 050040.9-045617 & $\mathrm{BD}-05^{\circ} 1122$ & $\mathrm{~F}^{\prime} \mathrm{V}^{f}$ & 9.94 & 0.52 & SB? & OAC(Dec. 03-Mar. 04) \\
\hline $050105.6+064209$ & & K3 & 12.60 & 1.03 & Single & OAC(Jan.-Mar. 02)/SPM 99 \\
\hline $050306.4+054851$ & & $\mathrm{G} 2 \mathrm{~V}^{f}$ & 10.62 & 0.65 & $\mathrm{SB} ?$ & OAC(Dec. 03-Mar. 04) \\
\hline $0503.8-1130$ & & $\mathrm{~K} 1$ & $12.26^{c}$ & & Single & SPM 99 \\
\hline $0507.8-0931$ & & $\mathrm{~K} 2$ & $12.50^{c}$ & $1.1:^{\ddagger}$ & SB2? & SPM 99/CA \\
\hline $0509.0-0315$ & & $\mathrm{~K} 1$ & $11.27^{g}$ & & Single & SPM 99 \\
\hline $0511.7-0348$ & & $\mathrm{~K} 1$ & $11.68^{\ddagger}$ & $1.3: \ddagger$ & Single & SPM 99 \\
\hline 051754.9-070819 & & $\mathrm{K} 0^{f}$ & 10.63 & 0.83 & Single & OAC(Dec. 03-Mar. 04) \\
\hline $0518.0+0712$ & & $\mathrm{~K} 2$ & 12.36 & 0.98 & SB? & OAC(Feb. 06)/SPM 99 \\
\hline $0518.6+0959$ & & $\mathrm{~K} 2$ & 12.47 & 0.83 & Single & OAC(Dec. 05-Feb. 06)/SPM 03 \\
\hline $052254.9+085803$ & & G9 & 10.28 & 0.88 & $\mathrm{SB} 2^{e}$ & OAC(Oct. 00-Feb. 01)/CA/SPM 03 \\
\hline $052345.0-075343$ & & $\mathrm{~K} 5 \mathrm{~V}^{f}$ & 12.24 & 1.20 & Single & OAC(Dec. 03-Mar. 04) \\
\hline $052423.1-064007$ & & G5III-IV ${ }^{f}$ & 12.24 & 1.01 & Single & OAC(Dec. 03-Mar. 04) \\
\hline $0528.0-0053$ & & K0 & $12.72^{c}$ & & SB2? & SPM 99/CA \\
\hline $052847.3+004840$ & & K0 & 12.86 & 0.81 & SB2? & OAC(Jan. 99-Feb. 01) $)^{\dagger} / \mathrm{CA}$ \\
\hline $052922.5+004112$ & V 1642 Ori & $\mathrm{K} 1 / \mathrm{K} 7$ & $12.35^{*}$ & $0.93^{*}$ & $\mathrm{~EB}-\mathrm{SB} 2^{d}$ & OAC(Jan. 99-Feb. 06 $)^{\dagger} / \mathrm{CA}$ \\
\hline $053005.6+004121$ & HD 290477 & $\mathrm{G} 3^{f}$ & 9.52 & 0.63 & Single & OAC(Dec. 00-Feb. 01, Oct. 05-Jan. 06) \\
\hline $053043.1-043453$ & Parenago 523 & $\mathrm{~K} 2-3 / \mathrm{K} 2-3^{d}$ & 11.36 & 1.11 & $\mathrm{SB} 2^{d}$ & $\mathrm{OAC}\left(\mathrm{Jan} .{ }^{99-F e b . ~ 01)}\right)^{\dagger} / \mathrm{CA}$ \\
\hline $053055.8+101506$ & LH98202 & $\mathrm{K} 2$ & 12.48 & 1.03 & SB? & OAC(Jan.-Mar. 02, Dec. 05-Feb. 06)/SPM 03 \\
\hline $053202.1-073153$ & & $\mathrm{~K} 2-3 / \mathrm{K} 3^{d}$ & 12.48 & 0.86 & $\mathrm{SB} 2^{d}$ & OAC(Oct. 00-Feb. 01)/CA/SPM 99 \\
\hline $0532.4+0131 \mathrm{a}$ & & $\mathrm{K} 2^{c}$ & $11.98^{c}$ & & Single? & SPM 99/03 \\
\hline $0532.4+0131 b$ & & $\mathrm{~K} 5^{c}$ & $13.78^{c}$ & & Single & SPM 99 \\
\hline $053231.8-042135$ & Parenago 930 & K3 & 11.83 & 1.00 & Single & OAC(Jan.-Mar. 02)/SPM 99 \\
\hline $053236.6-052254$ & HR Ori & K3 & 11.88 & 1.08 & Single? & OAC(Jan.-Mar. 02)/SPM 03 \\
\hline 053304.9-075850 & & $\mathrm{K} 1$ & 11.81 & 0.91 & Single & OAC(Oct. 00-Feb. 01)/SPM 03 \\
\hline $0533.1+0224$ & & K4 & $13.49^{c}$ & & SB? & SPM 03 \\
\hline $0534.7+1114$ & & K4 & $12.41^{c}$ & & Single & SPM 03 \\
\hline $0535.0-0411$ & Parenago 1679 & G9 & 11.97 & 0.91 & Single? & OAC(Oct. 00-Feb. 01)/CA/SPM 03 \\
\hline $053534.2-015227$ & & G6 & 11.89 & 0.67 & SB2? & OAC(Jan. 99-Mar. 00) ${ }^{\dagger}$, (oct. 05-feb. 06) \\
\hline $0536.7+0907$ & & $\mathrm{~K} 2$ & 12.37 & 1.01 & Single & OAC(Dec. 05-Feb. 06)/SPM 00 \\
\hline $0539.3+0918$ & & K1 & 11.44 & 0.95 & SB? & OAC(Oct. 00-Mar. 02) $)^{\dagger} /$ SPM 03 \\
\hline $0539.8-0205$ & & K6 & 12.94 & 1.14 & $\mathrm{SB} 2^{e}$ & OAC(Dec. 99-Feb. 00, feb. 06) \\
\hline $053956.8+095638$ & & $\mathrm{~K} 2$ & 10.70 & 0.89 & SB1 & OAC(Dec. 99-Feb. 01) ${ }^{\dagger}$ \\
\hline 054014.0-070832 & & K0 & $12.4:^{\ddagger}$ & $0.9:^{\ddagger}$ & SB2? & $\mathrm{CA}$ \\
\hline $054033.2-012157$ & & K1 & 11.93 & 0.87 & SB2? & OAC(Jan. 99-Mar. 00) ${ }^{\dagger} / \mathrm{CA} / \mathrm{SPM} 03$ \\
\hline $054124.0-032446$ & & $\mathrm{G} 8 / \mathrm{K} 3^{d}$ & 11.66 & 0.86 & $\mathrm{SB} 2^{d}$ & OAC(Jan. 99-Mar. 00) $)^{\dagger} / \mathrm{CA}$ \\
\hline $0544.6-0121$ & V523 Ori & $\mathrm{K} 1 \mathrm{~V}^{b}$ & 13.3: & 1.3: & Single & OAC(Feb. 06)/SPM 00 \\
\hline
\end{tabular}

* Out of eclipse; ${ }^{\dagger}$ more observing seasons; ${ }^{\ddagger}$ from Tycho Catalogue or Simbad.

${ }^{a}$ Alcalá et al. (2000a); ${ }^{b}$ present work; ${ }^{c}$ Chavarría-K et al. (2000); ${ }^{d}$ Covino et al. (2001); ${ }^{e}$ G. Torres, private communication; ${ }^{f}$ Frasca et al. (2003); ${ }^{g}$ Alcalá et al. (1996).

photometric errors, estimated from measurements of standard stars with a brightness comparable to the program stars, are typically $\sigma_{V}=0.01$ and $\sigma_{B-V}=0.02$. The monitoring frequency was variable from one run to another, but all the thirty program stars of the OAC sample were normally observed many times per night.

In addition, CCD photometry in the $B$ and $V$ Johnson filters was carried out at the $1.5 \mathrm{~m}$ telescope at the Estación de Observación de Calar Alto (CA, Spain) from December 3 to 13,1998 . We used a Tektronics CCD (TK1024AB), $1024 \times$ 1024 pixels, with a $24 \mu \mathrm{m}$ pixel size $\left(6.9 \times 6.9 \mathrm{arcmin}^{2}\right.$ field $)$. Integration times varied between 30 and $240 \mathrm{~s}$, in order to get $2 / 3$ of the saturation value at the maximum intensity pixel for the brightest target or comparison star in the field. Fourteen stars were observed at CA with a monitoring frequency of typically four times per night with two pointings done $3-4 \mathrm{~h}$ apart. The first two nights and the ninth night were lost due to bad weather conditions.
Further CCD photometric runs, in the $V$ and $R$ Johnson filters, were also performed from February 22 to March 4, 1999, from March 24 to 31, 2000, and from January 10 to 17, 2003, with the $1.5 \mathrm{~m}$ and $0.84 \mathrm{~m}$ telescopes of the San Pedro Mártir (SPM) Observatory, Baja California, Mexico (2790 m a.s.l). The CCDs employed were a TH7398M of $2048 \times 2048$ pixels (size $14 \times 14 \mu \mathrm{m}$ ) at the $1.5 \mathrm{~m}$ telescope and a SI003 of $1024 \times 1024$ pixels (size $24 \times 24 \mu \mathrm{m}$ ) at the $0.84 \mathrm{~m}$ telescope. The latter CCD has a Metachrome II and VISAR coating to improve its efficiency in the blue. Twenty-three targets were observed once per night or twice per night, at most, during the three observing runs at SPM.

A concise log indicating the site and season of the observations is given in the last column of Table 1.

The CCD data were reduced using the standard steps for CCD images reduction, including bias subtraction and division by an average normalized flat-field, obtained from twilight sky images. 
Aperture photometry for all the stars present in the images and having a signal-to-noise ratio $S / N \geq 100$ was performed with DAOPHOT under IRAF ${ }^{1}$. We calculated the differential magnitude of each target with respect to an artificial comparison star whose magnitude was built up with the counts of all the non-variable stars in the same field of view of the CCD detector, following the guidelines of Broeg et al. (2005).

\section{Time-series analysis}

We derived the periods of light variations by applying to the $V$ and $B$ magnitudes a periodogram analysis (Scargle 1982) and the CLEAN deconvolution algorithm (Roberts et al. 1987), which allowed us to reject aliases generated by the incompleteness of the data sampling and by the finite time-span of the observations. For each source, the highest peak was selected in the cleaned power spectrum of the $V$ light-curve (LC). Whenever the same period was found in the periodogram of the other band ( $B$ for the OAC and CA, $R$ for the SPM observations), without other peaks comparable in intensity with the main one, that period was considered as reliable. In the opposite case, the period was considered only as a "possible" one and is marked with a colon in Table 2. For one source, namely RX J0534.7+1114, we were not able to detect any periodicity, due to the limited time span and the low amplitude of magnitude variation. Thus, in total, we determined the rotation periods for 39 stars. False-alarm probabilities (FAP) for the peaks in the periodograms were also computed according to the definitions given by Scargle (1982) and by Horne \& Baliunas (1986). With the exception of only five stars, the periods from OAC data have been detected with a confidence level (1-FAP) $>99 \%$. Four stars have a confidence level between $90 \%$ and $99 \%$ and only one (1RXS J050105.6+064209) has an uncertain period detected with a confidence level of only $80 \%$. The periods determined from CA data have a confidence level always greater than $98 \%$. However, as stressed by Horne \& Baliunas (1986), the FAP depends on the data sampling. Thus, the actual false alarm probability might be smaller (higher confidence) for severely unevenly sampled data, but it might also be higher (lower confidence) due to data points taken too close together that are not really independent and tend to inflate the power artificially. For the SPM photometry the confidence levels are lower and typically in the range 80-95\%. This could be due to the few observing nights and the limited time span, but it could be also the result of a more regular data sampling.

The LC collection for OAC data is displayed in Fig. 1, where the source names and the photometric periods are indicated. The LCs obtained at SPM and CA are shown in Figs. 2 and 3, respectively.

\section{Results}

The measured rotational periods and the amplitudes of LCs in the $V$ filter, as well as the orbital periods for spectroscopic binaries, are reported in Table 2. Some physical parameters, such as $v \sin i, \mathrm{X}$-ray luminosity, and lithium equivalent width given in previous works (Alcalá et al. 1996, 2000a; Covino et al. 2001; Frasca et al. 2003), are also reported. For three objects, observed

${ }^{1}$ IRAF is distributed by the National Optical Astronomy Observatory, which is operated by the Association of the Universities for Research in Astronomy, inc. (AURA) under cooperative agreement with the National Science Foundation.

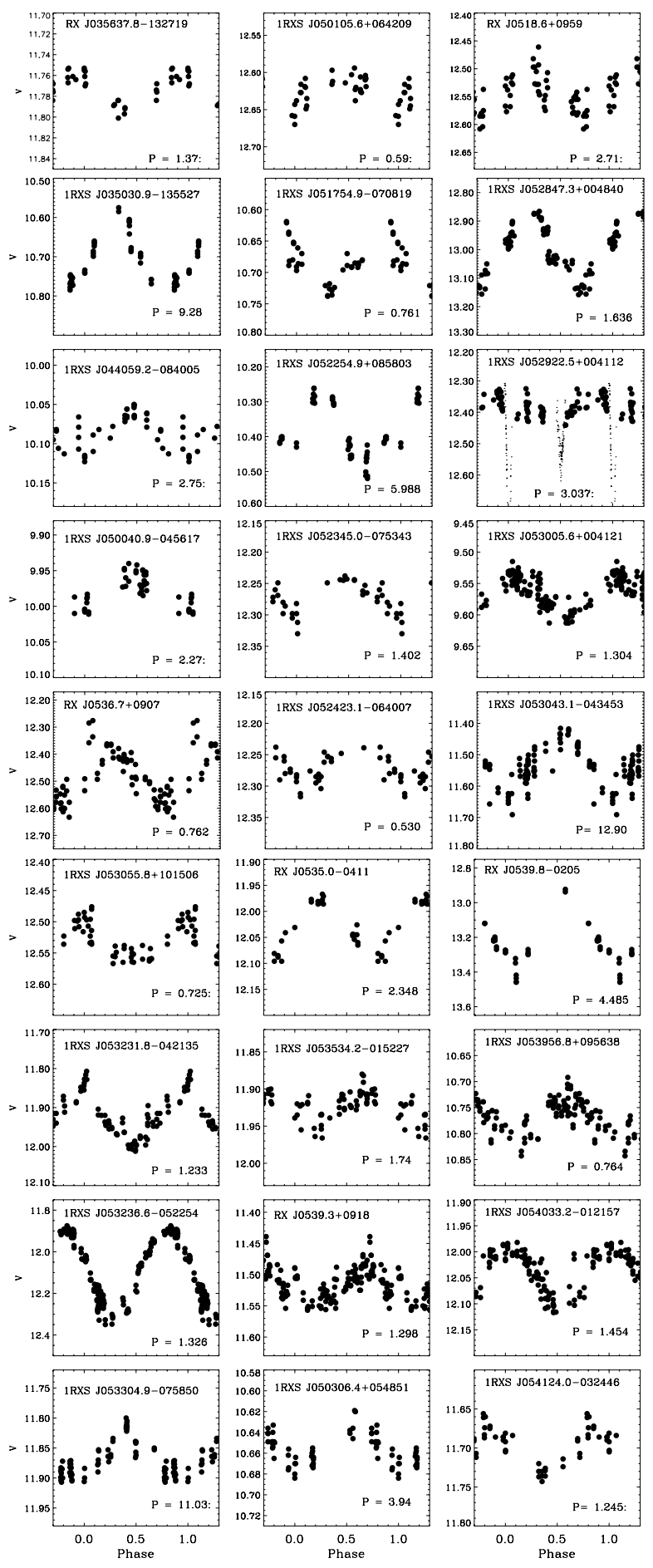

Fig. 1. $V$ light-curves obtained at OAC. Source names and photometric periods in days are indicated. The in-eclipse data points of 1RXS J052922.5+004112 are displayed with small dots.

only at low resolution, the lithium equivalent width could not be measured.

Eight of our targets are double-lined spectroscopic binaries (SB2) with orbital parameters derived from high-resolution spectroscopy (Covino et al. 2001; G. Torres, private communication). One of them, namely 1 RXS J052922.5+004112, is also an eclipsing binary (Covino et al. 2000, 2004). Twelve objects are also reported by Alcalá et al. (2000a) or by Frasca et al. (2003) as possible spectroscopic binaries on the basis of some indication of variable radial velocity $(\mathrm{RV})$ or due to a complex shape 

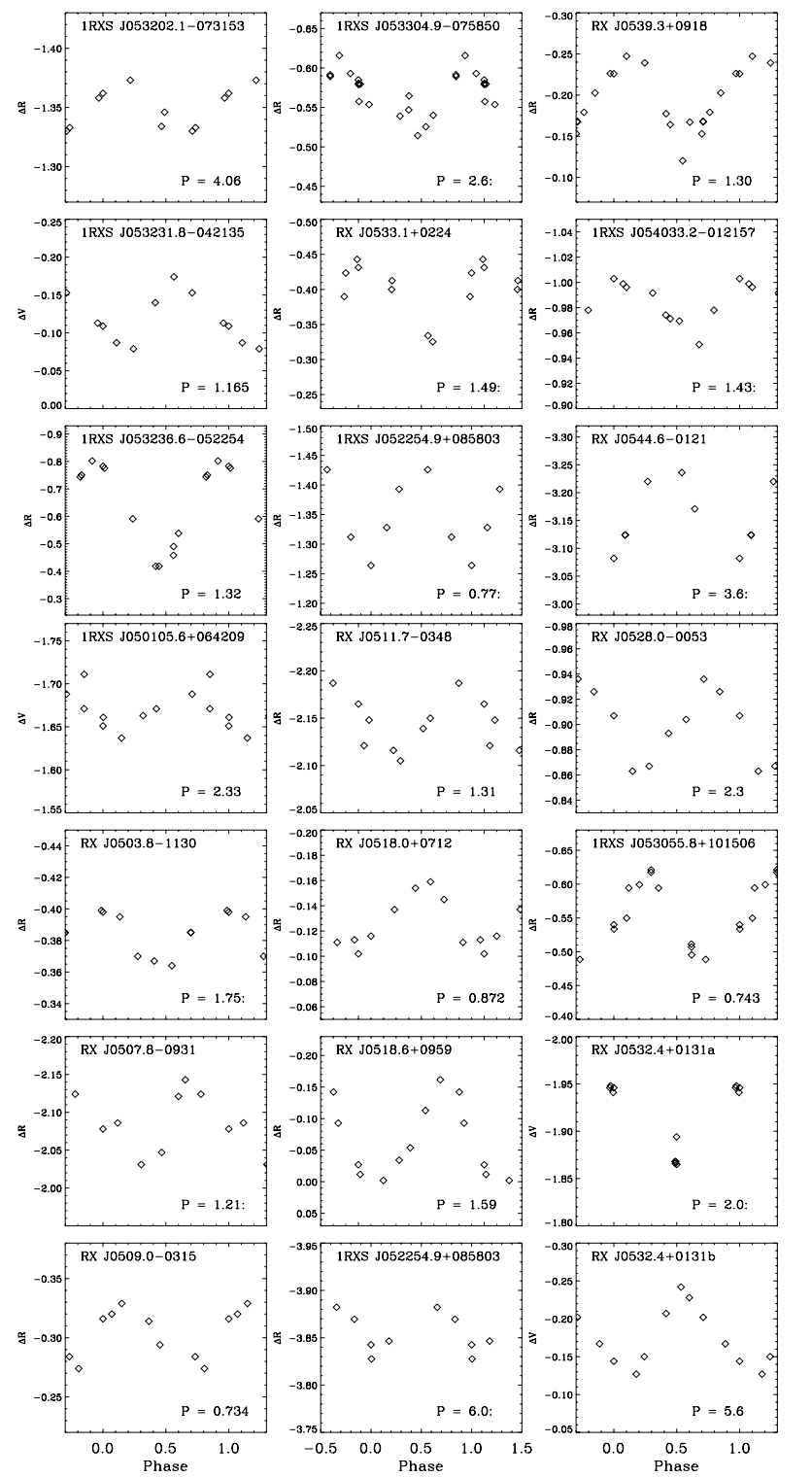

Fig. 2. Differential SPM light-curves for all the stars with detected rotational periods. The object identifier and corresponding photometric period (in days) are indicated.

of the cross-correlation function (CCF) showing asymmetric or multiple peaks. In very fast rotators, the measurement of RV is strongly hampered by the large width of the CCF and by the line distortions due to starspots, that can also mimic the presence of two or more blended components. For this reason, without additional spectra, we cannot firmly establish the binary nature of these twelve candidates.

The star 1 RXSJ $053956.8+095638$ is reported as a singlelined (SB1) spectroscopic binary (Alcalá et al. 2000a), but no RV curve has been derived so far, although there is an indication for a very long orbital period (G. Torres, private communication). The very short rotation period $(0.76)$ could be symptomatic of an evolved low-mass WTTS approaching the ZAMS with a steady increase of the rotation rate during its contraction along the evolutionary track.

The remaining objects of the sample do not show any evidence for the presence of close companions (either visual or spectroscopic), thus they will be treated here as single stars.

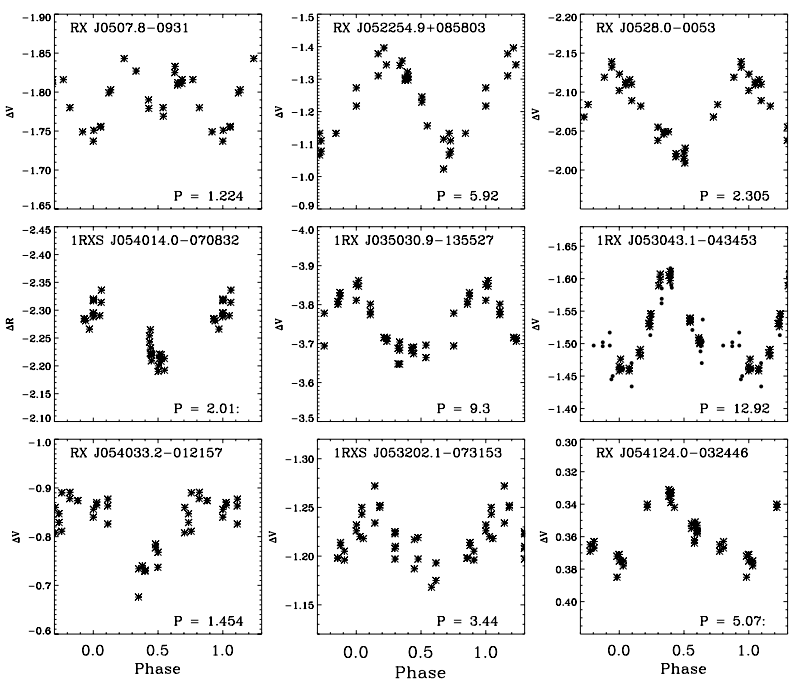

Fig. 3. Differential CA light-curves (asterisks) for all the stars with detected rotational periods. Source names and photometric periods (in days) are indicated. For 1RXS J053043.1-043453, the differential $V$ magnitudes obtained at OAC one month later are also displayed with small dots.

\subsection{Stellar parameters}

The effective temperatures were derived from the spectral types by means of the calibration proposed by de Jager \& Nieuwenhuijzen (1987). With an uncertainty of 1-2 spectral subclasses, we estimate a temperature error of the order of $150 \mathrm{~K}$. For the presumably single stars and for the SB1 systems in our sample, the stellar luminosity was determined by means of the observed spectral energy distribution (SED) obtained from the $U B V R_{\mathrm{C}} I_{\mathrm{C}} J H K L$ magnitudes. The $B$ and $V$ magnitudes corresponding to the maximum brightness (minimum spot visibility) in the light-curves obtained at OAC were used. For nearly all the stars observed at OAC, we obtained also a value of the $U$ magnitude. $R_{\mathrm{C}}$ and $I_{\mathrm{C}}$ magnitudes reported by Alcalá et al. (1996) and $J H K$ data coming from the 2MASS catalogue (Cutri et al. 2003) or from Alcalá et al. (1996) were used to complete the SEDs. For a few objects, $L$ magnitudes are also reported in the latter paper. We then used the grid of NextGen low-resolution synthetic spectra with $\log g=3.5$ and solar metallicity by Hauschildt et al. (1999) to perform a twoparameter fit of the SEDs. We assumed the mean distance to the Orion complex ( $d=460 \mathrm{pc}$, Genzel \& Stutzki 1989) to convert the apparent magnitudes into absolute ones, with the exceptions of the few objects clearly associated with Ori OB1a (see Table 3), for which the distance of $336 \mathrm{pc}$ was adopted (de Zeeuw et al. 1999). In both cases, we assumed a $20 \%$ error on the distance. For each star, we fixed the effective temperature of the NextGen spectrum closer to that derived from the spectral classification and we let the extinction, $A_{V}$, and the stellar radius, $R_{*}$, free to vary. The best solution was found by minimizing the $\chi^{2}$ of the fit. Figure 4 displays an example of the fitting procedure. The stellar luminosity was then obtained by integrating the best-fit model spectrum.

The $A_{V}$ values, listed in Table 3, are fairly consistent with the determinations made by Frasca et al. (2003) for some of these stars from the $B-V$ vs $U-B$ diagram and with the typical value, $A_{V} \approx 0.3 \mathrm{mag}$, expected from a standard extinction of $0.7-0.8 \mathrm{mag} \mathrm{kpc}^{-1}$ at the mean Orion distance $(460 \mathrm{pc})$. We have only four exceptions, with $A_{V}$ values around 1 mag. RX J0544.6-0121, one of the faintest objects in our sample, 
Table 2. Derived $P_{\text {rot }}$ and other physical quantities of our targets. A colon marks uncertain periods.

\begin{tabular}{|c|c|c|c|c|c|c|c|c|c|c|}
\hline 1RXS J/RX J & $\begin{array}{l}\text { Rate } \\
\left(\text { count s}^{-1}\right)\end{array}$ & $\begin{array}{l}\log L_{\mathrm{X}}{ }^{c} \\
\left(\mathrm{erg} \mathrm{cm}^{-2} \mathrm{~s}^{-1}\right)\end{array}$ & $\begin{array}{l}W(\mathrm{Li}) \\
(\mathrm{m} \AA)\end{array}$ & $\begin{array}{l}v \sin i \\
\left(\mathrm{~km} \mathrm{~s}^{-1}\right)\end{array}$ & $\begin{array}{l}\Delta V \\
(\mathrm{mag})\end{array}$ & $\begin{array}{l}P_{\text {rot }}^{\mathrm{OAC}} \\
\text { (days) }\end{array}$ & $\begin{array}{l}P_{\text {rot }}^{\mathrm{CA}} \\
\text { (days) }\end{array}$ & $\begin{array}{l}P_{\text {rot }}^{\text {SPM }} \\
\text { (days) }\end{array}$ & $\begin{array}{l}P_{\text {orb }} \\
\text { (days) }\end{array}$ & $e$ \\
\hline $035030.9-135527$ & $0.055 \pm 0.012$ & $31.22_{-0.26}^{+0.16}$ & $210 / 250^{a}$ & $19 / 7^{a}$ & 0.19 & 9.34 & 9.3 & - & $9.28^{a}$ & $0.00^{a}$ \\
\hline $035637.8-132719$ & $0.053 \pm 0.016$ & $31.21_{-0.30}^{+0.18}$ & $400^{e}$ & $80^{e}$ & 0.04 & 1.37: & - & - & - & - \\
\hline 044059.2-084005 & $0.048 \pm 0.011$ & $31.16_{-0.27}^{+0.16}$ & $200 / 135^{a}$ & $2 / 1^{a}$ & 0.06 & 2.75: & - & - & $13.56^{a}$ & $0.22^{a}$ \\
\hline 050040.9-045617 & $0.087 \pm 0.020$ & $31.42_{-0.27}^{+0.16}$ & - & $60:^{b}$ & 0.06 & $2.27:$ & - & - & - & - \\
\hline $050105.6+064209$ & $0.043 \pm 0.013$ & $31.11_{-0.30}^{+0.18}$ & $400^{c}$ & $21^{c}$ & 0.07 & $0.59:$ & - & 2.33 & - & - \\
\hline $050306.4+054851$ & $0.051 \pm 0.015$ & $31.19_{-0.30}^{+0.30}$ & - & $80:^{b}$ & 0.05 & 3.94 & - & - & - & - \\
\hline $0503.8-1130$ & $0.057 \pm 0.016$ & $31.24_{-0.29}^{+0.17}$ & $360^{c}$ & $25^{c}$ & 0.04 & - & - & 1.75: & - & - \\
\hline $0507.8-0931$ & $0.045 \pm 0.015$ & $31.13_{-0.12}^{-0.29}$ & $330^{c}$ & $64^{c}$ & 0.11 & - & 1.224 & 1.21: & - & - \\
\hline $0509.0-0315$ & $0.060 \pm 0.016$ & $31.26^{-0.172}$ & $320^{c}$ & $41^{c}$ & 0.05 & - & - & 0.734 & - & - \\
\hline 0511.7-0348 & $0.029 \pm 0.017$ & $30.94_{-0.54}^{-0.28}$ & $370^{c}$ & $35^{c}$ & 0.08 & - & - & 1.31 & - & - \\
\hline 051754.9-070819 & $0.123 \pm 0.048$ & $31.57_{-0.36}^{-0.54}$ & $260^{c}$ & $17^{c}$ & 0.09 & 0.761 & - & - & - & - \\
\hline $0518.0+0712$ & $0.034 \pm 0.016$ & $31.01_{-0.42}^{-0.21}$ & $410^{c}$ & $74^{c}$ & 0.06 & - & - & 0.872 & - & - \\
\hline $0518.6+0959$ & $0.023 \pm 0.012$ & $30.84_{-0.47}^{+0.22}$ & $370^{c}$ & $42^{c}$ & 0.18 & 2.71: & - & 1.59 & - & - \\
\hline $052254.9+085803$ & $0.261 \pm 0.042$ & $31.90_{-0.25}^{+0.16}$ & $200^{d}$ & - & 0.25 & 5.988 & 5.92 & 6: & $5.935^{\dagger}$ & $0.00^{\dagger}$ \\
\hline $052345.0-075343$ & $0.145 \pm 0.029$ & $31.64_{-0.26}^{+0.16}$ & - & $<45:^{b}$ & 0.07 & 1.402 & - & - & - & - \\
\hline $052423.1-064007$ & $0.025 \pm 0.016$ & $30.88_{-0.61}^{+0.26}$ & $410^{b}$ & $52^{b}$ & 0.07 & 0.530 & - & - & - & - \\
\hline 0528.0-0053 & $0.028 \pm 0.014$ & $30.66_{-0.44}^{+0.01}$ & $300^{d}$ & - & 0.13 & - & 2.305 & 2.3 & - & - \\
\hline $052847.3+004840$ & $0.032 \pm 0.014$ & $30.71_{-0.39}^{+0.20}$ & $280^{d}$ & - & 0.28 & 1.636 & - & - & - & - \\
\hline $052922.5+004112$ & $0.048 \pm 0.017$ & $30.86_{-0.33}^{+0.19}$ & $370 / 450^{a}$ & $25 / 17^{a}$ & $0.12^{*}$ & 3.032: & - & - & 3.037 & 0.00 \\
\hline $053005.6+004121$ & $0.193 \pm 0.030$ & $31.49_{-0.24}^{+0.16}$ & $260^{c}$ & $60^{c}$ & 0.09 & 1.304 & - & - & - & - \\
\hline $053043.1-043453$ & $0.050 \pm 0.018$ & $31.18_{-0.34}^{+0.19}$ & $580 / 580^{a}$ & $13 / 13^{a}$ & 0.22 & 12.90 & $>9-12.92^{\star}$ & - & $40.57^{a}$ & $0.32^{a}$ \\
\hline $053055.8+101506$ & $0.067 \pm 0.019$ & $31.31_{-0.29}^{-0.17}$ & $520^{d}$ & $150^{c}$ & 0.08 & $0.725:$ & - & 0.743 & - & - \\
\hline $053202.1-073153$ & $0.023 \pm 0.014$ & $30.84_{-0.57}^{+0.29}$ & $490 / 470^{a}$ & $20 / 22^{a}$ & 0.10 & - & 3.44 & 4.06: & $46.85^{a}$ & $0.47^{a}$ \\
\hline $0532.4+0131 \mathrm{a}$ & $0.025 \pm 0.013^{\ddagger}$ & $30.61_{-0.46}^{+0.21}$ & $365^{c}$ & $23^{c}$ & 0.07: & - & - & $2.0:$ & - & - \\
\hline $0532.4+0131 b$ & - & - & $460^{c}$ & $17^{c}$ & 0.11 & - & - & 5.6 & - & - \\
\hline $053231.8-042135$ & $0.032 \pm 0.014$ & $30.99_{-0.39}^{+0.20}$ & $495^{c}$ & $21^{c}$ & 0.17 & 1.233 & - & 1.165 & - & - \\
\hline $053236.6-052254$ & $0.120 \pm 0.024$ & $31.56_{-0.26}^{+0.16}$ & $500^{c}$ & $125^{c}$ & 0.45 & 1.326 & - & 1.32 & - & - \\
\hline 053304.9-075850 & $0.082 \pm 0.028$ & $31.39_{-0.32}^{+0.18}$ & $420^{c}$ & $47^{c}$ & 0.09 & 11.03: & - & 2.6: & - & - \\
\hline $0533.1+0224$ & $0.038 \pm 0.015$ & $31.06_{-0.36}^{+0.19}$ & $440^{c}$ & $54^{c}$ & 0.12 : & - & - & 1.49: & - & - \\
\hline $0534.7+1114$ & $0.023 \pm 0.015$ & $30.84_{-0.63}^{+0.25}$ & $280^{c}$ & $20^{c}$ & - & - & - & - & - & - \\
\hline 0535.0-0411 & $0.038 \pm 0.014$ & $31.06_{-0.34}^{+0.0 .19}$ & $360^{c}$ & $20^{c}$ & 0.12 & 2.348 & - & - & - & - \\
\hline $053534.2-015227$ & $0.018 \pm 0.011$ & $30.74_{-0.57}^{+0.24}$ & $320^{d}$ & - & 0.06 & 1.74 & - & - & - & - \\
\hline $0536.7+0907$ & $0.049 \pm 0.014$ & $31.17_{-0.29}^{+0.17}$ & $455^{c}$ & $28^{c}$ & 0.22 & 0.762 & - & $0.77:$ & - & - \\
\hline $0539.3+0918$ & $0.044 \pm 0.014$ & $31.12_{-0.31}^{+0.18}$ & $400^{c}$ & $108^{c}, 54^{\dagger}$ & 0.09 & 1.298 & - & 1.30 & - & - \\
\hline 0539.8-0205 & $0.052 \pm 0.014$ & $31.20_{-0.29}^{+0.11}$ & $530^{d}$ & - & 0.40 & 4.485 & - & - & $18.74^{\dagger}$ & $0.39^{\dagger}$ \\
\hline $053956.8+095638$ & $0.377 \pm 0.036$ & $32.06_{-0.23}^{+0.15}$ & $310^{d}$ & $64^{\dagger}$ & 0.13 & 0.764 & - & - & - & - \\
\hline $054014.0-070832$ & $0.033 \pm 0.013$ & $31.00_{-0.36}^{+0.19}$ & $250^{c}$ & $36^{c}$ & 0.13 & - & 2.01: & - & - & - \\
\hline $054033.2-012157$ & $0.048 \pm 0.013$ & $31.16_{-029}^{+0.17}$ & $400^{d}$ & - & 0.17 & 1.454 & 1.454 & 1.43: & - & - \\
\hline $054124.0-032446$ & $0.049 \pm 0.013$ & $31.17_{-0.28}^{+0.17}$ & $320 / 400^{a}$ & $22 / 15^{a}$ & 0.06 & 1.245: & $5.07:-5.76^{\star}$ & - & $4.99^{a}$ & $0.00^{a}$ \\
\hline 0544.6-0121 & $0.052 \pm 0.013$ & $31.20_{-0.28}^{+0.17}$ & $405^{c}$ & $18^{e}$ & 0.15 : & - & - & 3.6: & - & - \\
\hline
\end{tabular}

* Out of eclipse (Oct. 05-Feb. 06 season); ${ }^{\star} \mathrm{OAC}$ and CA observations; ${ }^{\dagger}$ G. Torres, private communication; ${ }^{\ddagger}$ total $L_{\mathrm{X}}(a+b) ;:$ uncertain value.

${ }^{a}$ Covino et al. (2001); ${ }^{b}$ Frasca et al. (2003); ${ }^{c}$ Alcalá et al. (2000a); ${ }^{d}$ Alcalá et al. (1996); ${ }^{e}$ present work.

displays the most peculiar extinction, $A_{V}=3.3 \mathrm{mag}$, that could be the result of the relatively large photometric errors. However, this object is clearly surrounded by a reflection nebula and could be indeed a very reddened star. Extinction values of $\approx 1$ mag and ranging from about 0.5 to 2 mag were found by Greve et al. (1994) by measuring the ratios of emission lines in the Orion Nebula. Thus, the high values of $A_{V}$ we found for a few objects in our sample could be indicative of stars more deeply embedded into nebular matter.

The accuracy of the radii determinations mainly depends on the uncertainties on the distance and $T_{\text {eff }}$. We estimate that, typically, the errors on the radii are of the order of $15 \%$.
From the values of the stellar radius, $v \sin i$, and rotation period, we could also estimate the inclination angle, $i$, of the rotation axis to the line of sight. The values of $\sin i$, summarized in Table 3, are always lower than 1, with the exception of only one object, 1RXS J050306.4+054851, a possible binary for which the $v \sin i$ value of $80 \mathrm{~km} \mathrm{~s}^{-1}$ could be the effect of blended spectroscopic components. For other six stars we deduce very low values of inclination $\left(\sin i<0.25\right.$, i.e. $i<15^{\circ}$ ) for which the detection of rotational modulation should be highly unlikely (nearly pole-on rotators). The sin $i$ values in these cases are written in italics in Table 3. Whenever two possible periods from different data sets, or two $v \sin i$ determinations, are available, we have listed both $\sin i$ estimates in Table 3 . 
Table 3. Physical parameters derived from the SED analysis and from the HR diagram.

\begin{tabular}{|c|c|c|c|c|c|c|c|c|c|}
\hline 1RXS J/RX J & $\begin{array}{l}\text { Dist } \\
(\mathrm{pc})\end{array}$ & $\begin{array}{l}T_{\text {eff }} \\
(\mathrm{K})\end{array}$ & $\begin{array}{l}L \\
\left(L_{\odot}\right)\end{array}$ & $\begin{array}{l}A_{V} \\
(\mathrm{mag})\end{array}$ & $\begin{array}{l}R \\
\left(R_{\odot}\right)\end{array}$ & $\begin{array}{l}M_{\mathrm{DM}}^{d} \\
\left(M_{\odot}\right)\end{array}$ & $\begin{array}{l}M_{\mathrm{PS}}^{f} \\
\left(M_{\odot}\right)\end{array}$ & $\begin{array}{l}\text { Age }^{f} \\
(\mathrm{Myr})\end{array}$ & $\sin i^{a}$ \\
\hline $035637.8-132719$ & 460 & 4990 & 6.1 & 0.31 & 3.6 & 1.7 & 2.00 & 1.0 & 0.60 \\
\hline $050040.9-045617$ & 460 & 6100 & 22.8 & 0.23 & 4.1 & 2.5 & 2.25 & 1.0 & 0.66 \\
\hline $050105.6+064209$ & 460 & 4690 & 3.4 & 0.45 & 2.7 & 0.9 & 1.75 & 1.5 & $0.09-0.36$ \\
\hline $050306.4+054851$ & 460 & 5790 & 14.3 & 0.26 & 3.5 & 2.5 & 2.25 & $\leq 1$ & 1.78 \\
\hline $0503.8-1130$ & 460 & 4990 & 3.0 & 0.24 & 2.1 & 1.2 & 1.75 & 2.5 & 0.41 \\
\hline $0507.8-0931$ & 460 & 4840 & 2.8 & 0.24 & 2.2 & 1.5 & 1.75 & 3.0 & 0.70 \\
\hline $0509.0-0315$ & 460 & 4990 & 6.6 & 0.16 & 3.2 & 1.7 & 2.25 & $\leq 1$ & 0.19 \\
\hline $0511.7-0348$ & 460 & 4990 & 4.1 & 0.23 & 2.5 & 1.5 & 1.75 & 2.5 & 0.36 \\
\hline 051754.9-070819 & 460 & 5150 & 14.1 & 0.23 & 4.3 & 2.5 & 2.50 & $\leq 1$ & 0.06 \\
\hline $0518.0+0712$ & 460 & 4840 & 3.4 & 0.23 & 2.7 & 1.2 & 1.75 & 3.0 & 0.47 \\
\hline $0518.6+0959$ & 460 & 4840 & 3.0 & 0.23 & 2.5 & 1.2 & 1.75 & 3.0 & $0.90-0.53$ \\
\hline $052345.0-075343$ & 460 & 4410 & 8.2 & 0.95 & 4.5 & 0.7 & 1.35 & $\leq 1$ & $\leq 0.28$ \\
\hline $052423.1-064007$ & 460 & 5100 & 4.0 & 0.40 & 2.5 & 1.7 & 1.75 & 4.0 & 0.22 \\
\hline $0528.0-0053$ & $336^{g}$ & 5150 & 1.6 & 0.65 & 1.4 & 1.4 & 1.20 & 10.0 & - \\
\hline $052847.3+004840$ & $336^{g}$ & 5150 & 0.8 & 0.16 & 1.1 & 1.1 & 1.00 & 15.0 & - \\
\hline $053005.6+004121$ & $336^{g}$ & 5710 & 18.8 & 0.19 & 4.0 & 1.0 & 2.25 & 1.5 & 0.39 \\
\hline $053055.8+101506$ & 460 & 4840 & 3.7 & 0.40 & 2.8 & 1.3 & 1.75 & 3.0 & $0.76-0.79$ \\
\hline $0532.4+0131 \mathrm{a}$ & $336^{g}$ & 4840 & 1.9 & 0.16 & 2.0 & 1.0 & 1.50 & 4.0 & 0.45 \\
\hline $0532.4+0131 b$ & $336^{g}$ & 4410 & 1.4 & 1.33 & 1.8 & 0.7 & 1.20 & 3.0 & 1.0 \\
\hline $053231.8-042135$ & 460 & 4690 & 6.8 & 0.48 & 3.8 & 1.0 & 2.00 & $\leq 1$ & 0.13 \\
\hline 053236.6-052254 & 460 & 4690 & 7.8 & 0.67 & 4.0 & 1.0 & 2.00 & $\leq 1$ & 0.82 \\
\hline 053304.9-075850 & 460 & 4990 & 5.7 & 0.31 & 2.9 & 1.7 & 2.00 & 1.0 & $3.5-0.83$ \\
\hline $0533.1+0224$ & 460 & 4540 & 2.7 & 1.19 & 2.4 & 0.7 & 1.50 & 1.5 & 0.66 \\
\hline $0534.7+1114$ & 460 & 4540 & 2.8 & 0.23 & 2.4 & 0.7 & 1.50 & 1.0 & - \\
\hline $0535.0-0411$ & 460 & 5230 & 5.7 & 0.55 & 2.7 & 2.0 & 2.00 & 2.5 & 0.34 \\
\hline $053534.2-015227$ & 460 & 5470 & 4.4 & 0.23 & 2.2 & 1.6 & 1.50 & 4.0 & - \\
\hline $0536.7+0907$ & 460 & 4840 & 4.0 & 0.39 & 2.9 & 1.3 & 2.00 & 1.0 & 0.15 \\
\hline $0539.3+0918$ & 460 & 4990 & 7.7 & 0.24 & 3.4 & 2.0 & 2.25 & $\leq 1$ & $0.82-0.41$ \\
\hline $053956.8+095638$ & 460 & 4840 & 15.9 & 0.23 & 5.8 & 1.7 & 2.50 & $\leq 1$ & 0.17 \\
\hline 054014.0-070832 & 460 & 5150 & 3.2 & 0.39 & 2.1 & 1.7 & 1.50 & 4.0 & 0.68 \\
\hline 054033.2-012157 & 460 & 4990 & 5.1 & 0.23 & 2.8 & 1.5 & 2.00 & 1.0 & - \\
\hline $0544.6-0121$ & 460 & 4990 & 22.1 & 3.00 & 5.8 & 2.0 & 2.25 & $\leq 1$ & 0.22 \\
\hline $052922.5+004112 \mathrm{~A} 1^{b}$ & 325 & 5200 & 1.37 & - & 1.44 & - & $1.27^{b}$ & 10.0 & 0.998 \\
\hline $052922.5+004112 \mathrm{~A} 2^{b}$ & 325 & 4220 & 0.52 & - & 1.35 & - & $0.93^{b}$ & 10.0 & 0.998 \\
\hline $053043.1-043453 \mathrm{~A}^{c}$ & 460 & 4770 & 5.35 & - & 3.40 & 1.2 & $2.0^{f}$ & 1.0 & 0.98 \\
\hline $053043.1-043453 \mathrm{~B}^{c}$ & 460 & 4770 & 5.35 & - & 3.40 & 1.2 & $2.0^{f}$ & 1.0 & 0.98 \\
\hline $053202.1-073153 \mathrm{~A}^{c}$ & 460 & 4770 & 1.53 & - & 1.82 & 1.0 & $1.4^{f}$ & 5.0 & 0.81 \\
\hline $053202.1-073153 \mathrm{~B}^{c}$ & 460 & 4690 & 1.47 & - & 1.84 & 1.0 & $1.4^{f}$ & 5.0 & 0.81 \\
\hline $054124.0-032446 \mathrm{~A}^{c}$ & 460 & 5310 & 4.70 & - & 2.57 & 2.1 & $1.9^{f}$ & 3.0 & 0.88 \\
\hline $054124.0-032446 \mathrm{~B}^{c}$ & 460 & 4690 & 1.20 & - & 1.66 & 0.9 & $1.3^{f}$ & 7.5 & 0.88 \\
\hline
\end{tabular}

${ }^{a}$ Unreliable sin $i$ values in italic. ${ }^{b}$ Covino et al. (2004). ${ }^{c}$ Covino et al. (2001). ${ }^{d}$ From D'Antona \& Mazzitelli (1997) evolutionary tracks. ${ }^{f}$ From Palla \& Stahler (1999) evolutionary tracks. ${ }^{g}$ Associated with Ori OB1a.

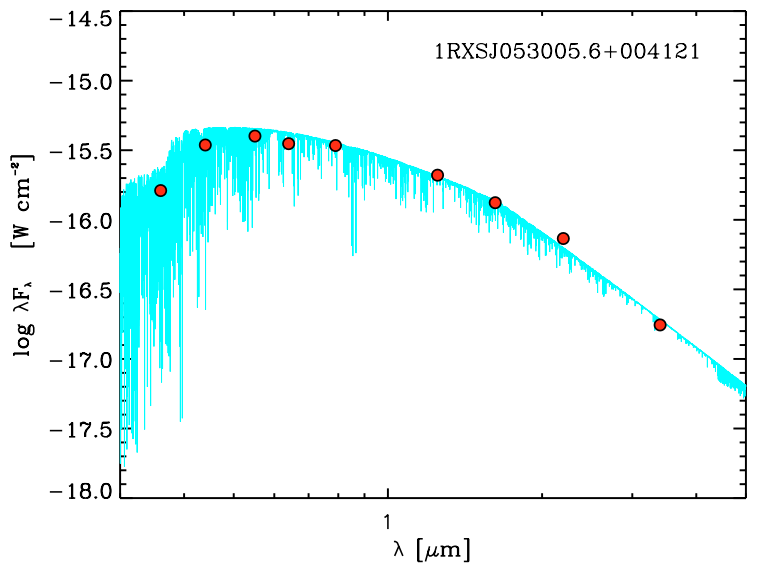

Fig. 4. Spectral energy distribution of 1RXS J053005.6+004121 obtained with the de-reddened $U B V R_{\mathrm{C}} I_{\mathrm{C}} J H K L$ magnitudes (dots). The best fitting NextGen spectrum is over-plotted.
Although the uncertainty on the object distance prevents us from deriving very accurate values for the luminosity, we located all the objects on the Hertzsprung-Russel (HR) diagram (Fig. 5) and roughly estimated their masses and ages by the comparison with the set of theoretical PMS tracks and isochrones by Palla \& Stahler (1999). The mass range of the investigated WTTS is about $1-2.5 M_{\odot}$. We also used the set of evolutionary PMS tracks by D'Antona \& Mazzitelli (1997) finding a slightly wider mass range $\left(0.7 M_{\odot} \leq M_{\mathrm{DM}} \leq 2.5 M_{\odot}\right)$. We observe an age spread of the WTTS in our sample which is significantly larger than that produced by the luminosity errors. We find that our sample contains both objects apparently younger that about $1 \mathrm{Myr}$ and older stars with ages up to $10-15 \mathrm{Myr}$.

Four out of the eight confirmed SB2's in our sample have orbital and physical parameters determined by Covino et al. (2001) or Covino et al. (2004). The components of these systems are also displayed in Fig. 5 with different symbols. 


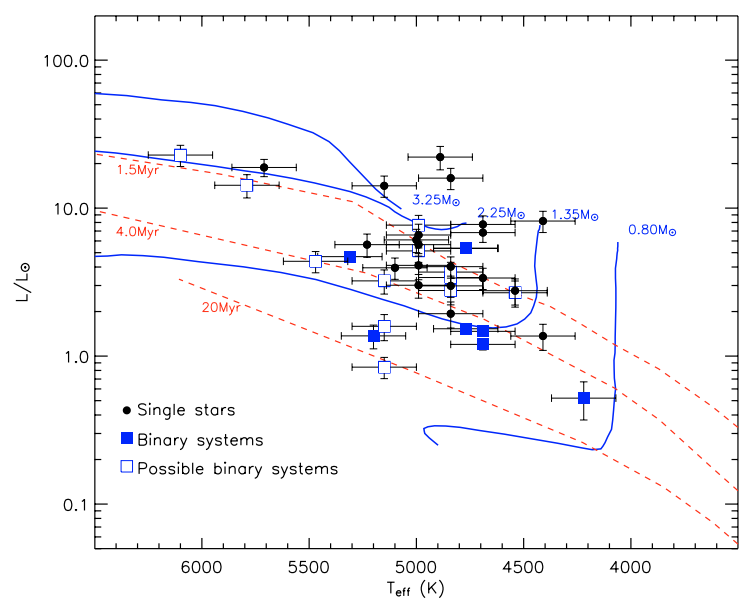

Fig. 5. HR diagram for the single and SB1 WTTS in our sample (dots) and for the components of the known SB2 systems (filled squares). Possible binaries are represented with open squares. The evolutionary PMS tracks and isochrones by Palla \& Stahler (1999) are also displayed in the figure by continuous and dotted lines, respectively.

\subsection{Rotation period distribution}

The rotation period distribution (Fig. 6a) of our targets appears nearly symmetric, with a median value $P_{\text {rot }} \simeq 1.7$. We did not include the $P_{\text {rot }}$ value of the binary 1RXS J035637.8-132719, because it is probably not a PMS star, as indicated by the moderate lithium content and by the circularization and synchronization attained at a relatively long orbital period (see Sect. 4.5). We also distinguished the certain from uncertain rotation periods (those labeled with colons in Table 2) in Fig. 6d by different shading on the histograms. However, the two distributions appear quite similar.

It is interesting to note that the long-period tail of the distribution is due only to the confirmed spectroscopic binaries, while the distribution of rotation periods for the single stars (including also the possible binaries) is slightly displaced towards shorter periods (see Fig. 6a) with a median value of $P_{\text {rot }} \simeq 1$ d.5. This does not necessarily mean that there are no slowly-rotating single stars among the sparse population of Orion WTTS, but they seem to be significantly less frequent then in the ONC. Moreover, the detection of long rotation periods only for the SBs implies that high-amplitude variations are observed only for the slowly-rotating components of close binaries (see Sect. 4.4).

No clear correlation between rotation period and age is found for the investigated stars. This might be due to the uncertainty on the individual ages deduced from the isochrones which can be as high as $5 \mathrm{Myr}$ as a consequence of the distance spread for such a large cloud. Indeed, evolutionary models are useful tools for determining the age of homogeneous and statistically significant star samples.

The rotation periods do not show any particular trend as a function of the $B-V$ color index in the spectral range spanned by our targets (F9-K6). This behavior confirms what already found for young open clusters (see, e.g., Marilli et al. 1997, and references therein). However, while for the young clusters the period distribution displays a clear separation between fast and slow rotators (see Figs. 6b,c), such bimodal behavior is not present in our sample. The period distributions for Pleiades $(\simeq 100 \mathrm{Myr})$ and $\alpha$ Persei $(\simeq 50 \mathrm{Myr})$, displayed with dashed histograms in Fig. 6b and Fig. 6c, respectively, were built using period determinations made or listed by Marilli et al. (1997); Rebull et al. (2004); Lamm et al. (2005); Pizzolato et al. (2003);
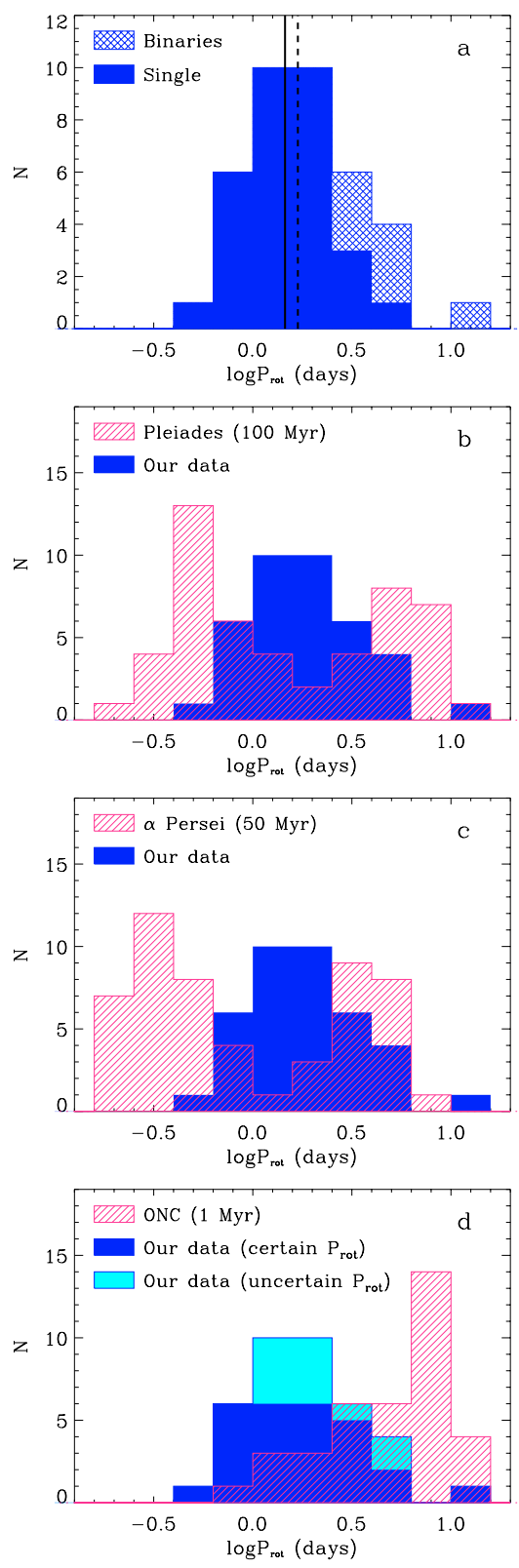

Fig. 6. a) Rotation period distribution for the WTTS in our sample. The hatched area refers to the confirmed binaries. The median of the distribution for the entire sample is marked by a dashed vertical line, while that for the presumably single stars is represented by the full line. b, c) The $\log \left(P_{\text {rot }}\right)$ distributions for all the Orion WTTS in our sample (filled histogram) is compared to that of stars in young open clusters (b Pleiades, c $\alpha$ Persei) built up with data taken from Marilli et al. (1997), Pizzolato et al. (2003), Rebull et al. (2004), Lamm et al. (2005), and Herbst \& Mundt (2005) and ONC members d) in the same mass range $\left(M>0.7 M_{\odot}\right)$ with periods measured by Herbst et al. (2002). In the lower panel, the distributions of stars with certain and uncertain periods (those ones with the colons in Table 2) in our sample are distinguished by different shading on the histograms.

Herbst \& Mundt (2005). In order to compare homogeneous samples, only stars with masses greater than about $0.7 M_{\odot}$ are considered.

It is apparent how the peaks of both the slow and fast rotators displace towards longer periods with the cluster age going from 50 to $100 \mathrm{Myr}$, due to the strong magnetic braking operating in these evolutionary stages.

We also compared the distribution of the rotation periods of our WTTS sample with that for the stars in the Orion Nebula 


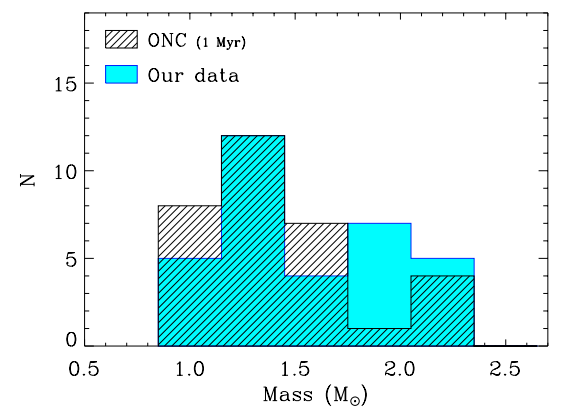

Fig. 7. Mass distributions for our targets (filled histogram) and for the stars in the Orion Nebula Cluster (ONC) with mass $M>0.7 M_{\odot}$ (hatched area) according to Herbst et al. (2002). For both star samples, the stellar masses have been evaluated from their position on the HR diagram and the evolutionary tracks of D'Antona \& Mazzitelli (1997).

Cluster (ONC, age $\approx 1$ Myr) determined by Herbst et al. (2002). Selecting stars in the same mass range of our targets $(M>$ $\left.0.7 M_{\odot}\right)$, a distribution peaked at $P_{\text {rot }} \simeq 7.1$, with a tail toward periods shorter than 1 day, is found. Its median value is $P_{\text {rot }} \simeq 6.6$.

The period difference between the peak centers of the two distributions could be due to:

i) an age effect on our sparse population of WTTS, with a possible larger age spread than the ONC members. The latter could be, on average, younger and still rotationally coupled to their own circumstellar disk (Bouvier et al. 1997; Rebull et al. 2006);

ii) an observational bias in our data toward the detection of shorter periods, due to the relatively short temporal base-line;

iii) a possible difference between the mass distributions of the two samples.

The choice of our targets as optical counterparts of X-ray sources might have preferentially selected stars with very high coronal emission, but this does not imply a strong bias towards faster rotators since for PMS stars there is almost no correlation between rotation and X-ray emission.

As far as the point (iii) is concerned, we remark that rotation may be mass-dependent over the range of masses included in our sample. For instance, Cohen et al. (2004) found that the G-type PMS stars in IC 348 rotate faster than the K5-M2 stars. Thus, if our targets have a mass distribution markedly different from the subsample of ONC stars with $M>0.7 M_{\odot}$, this could affect the observed period distribution. The mass distribution of the two samples is shown in Fig. 7. We built the histogram for our WTTS sample by using the masses derived from the D'Antona \& Mazzitelli (1997) evolutionary models in order to be consistent with the mass determinations for the ONC members obtained by Herbst et al. (2002). No significant difference between the two distributions is evident.

In any case these statistical distributions can contribute to clarify the evolution of rotation in solar-like stars from the PMS to the MS phase, when the effects of the magnetic disk-locking and of the braking induced by magnetized winds reduce the specific angular momentum by a factor $10-100$.

\subsection{The $X$-ray luminosity}

The X-ray luminosity for each object was determined assuming isotropic radiation as $L_{\mathrm{X}}=4 \pi d^{2} f_{\mathrm{X}}$, where $d$ is the distance and $f_{\mathrm{X}}$ is the $\mathrm{X}$-ray flux at the earth in $\mathrm{erg} \mathrm{s}^{-1} \mathrm{~cm}^{-2}$. The

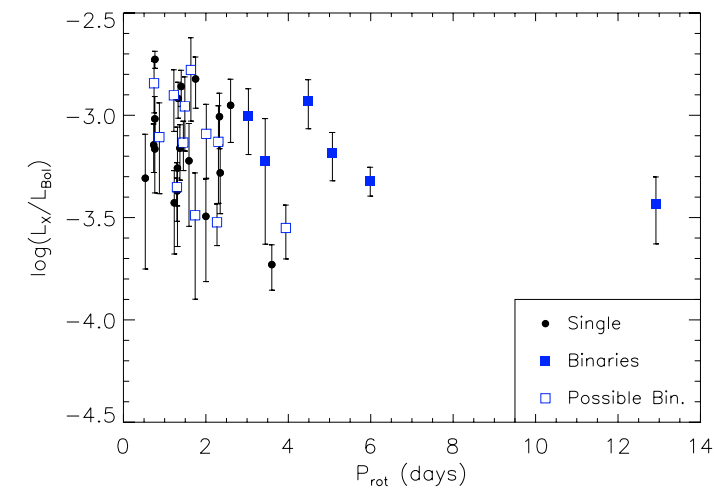

Fig. 8. $\log \left(L_{\mathrm{X}} / L_{\text {bol }}\right)$ versus $P_{\text {rot }}$ for the sources in our sample.

latter is given by $f_{\mathrm{X}}=E C F \cdot Z$, where $E C F$ is the energy conversion factor and $Z$ is the background and vignetting corrected broad-band $X$-ray count rate. We assume an average value of $1.2 \times 10^{-11} \mathrm{erg} \mathrm{cm}^{-2}$ count $^{-1}$ for $E C F$, which corresponds to a Raymond-Smith photon energy distribution with temperature in the range $10^{6}-10^{7} \mathrm{~K}$ in the ROSAT hard-energy band 0.5-2.5 keV (Neuhäuser et al. 1995; Alcalá et al. 2000b). The relative error on $L_{\mathrm{X}}$ was evaluated as

$\sigma_{L_{X}} / L_{X}=\sqrt{\left(\sigma_{Z} / Z\right)^{2}+\left(2 \sigma_{d} / d\right)^{2}}$

where $\sigma_{Z} / Z$ and $\sigma_{d} / d$ (assumed to be 0.2 , see Sect. 4.1) are the relative errors on the count rate and on the distance, respectively. The X-ray luminosities are presented in Table 2.

Nearly all the single and binary stars in our sample display a level of X-ray luminosity close to saturation $\left(\log L_{X}\right.$ in the range 30.6-32.1), with an apparent distribution independent of the rotation period. The luminosity ratio $\log \left(L_{\mathrm{X}} / L_{\text {bol }}\right)$ (Fig. 8) reaches a maximum value of about -2.7 , comparable to the value of -2.6 found for the WTTS in the Chamaeleon SFR (Alcala et al. 1997), rotating faster than about two days. In this period bin $\left(P_{\text {rot }}<2\right.$ days $)$ the highest spread of luminosity ratios is also observed. The data suggest a weak correlation of the coronal activity level with the rotation period, with a linear Pearson's correlation coefficient $\rho=-0.24$.

\subsection{Light-curve amplitudes}

The light-curve amplitudes do not show any definite trend as a function of $P_{\text {rot }}$ (Fig. 9a). The presence of both large and small amplitudes among the fast rotators could be due either to intrinsic effects, like magnetic saturation (Randich 1998), or to projection effects. Relatively large amplitudes for $P_{\text {rot }}$ greater than about 4 days are observed only in four binaries, the only longperiod objects for which we detected rotational modulation in the photometric time series.

The LC amplitudes show instead a scattered correlation with $T_{\text {eff }}$ or $B-V$, i.e. cooler stars tend to have a wider range of amplitudes (Fig. 9b), reaching values as high as $0.4-0$. 5 . An analogous behavior was found by Messina et al. (2003) for the very active main-sequence stars belonging to young open clusters, where higher amplitudes of the modulation are associated to later spectral types.

\subsection{Synchronization and circularization}

All the four systems with eccentricity $e \simeq 0$ appear to be also tidally synchronized (Fig. 10). Three of these systems have $P_{\text {orb }}$ 

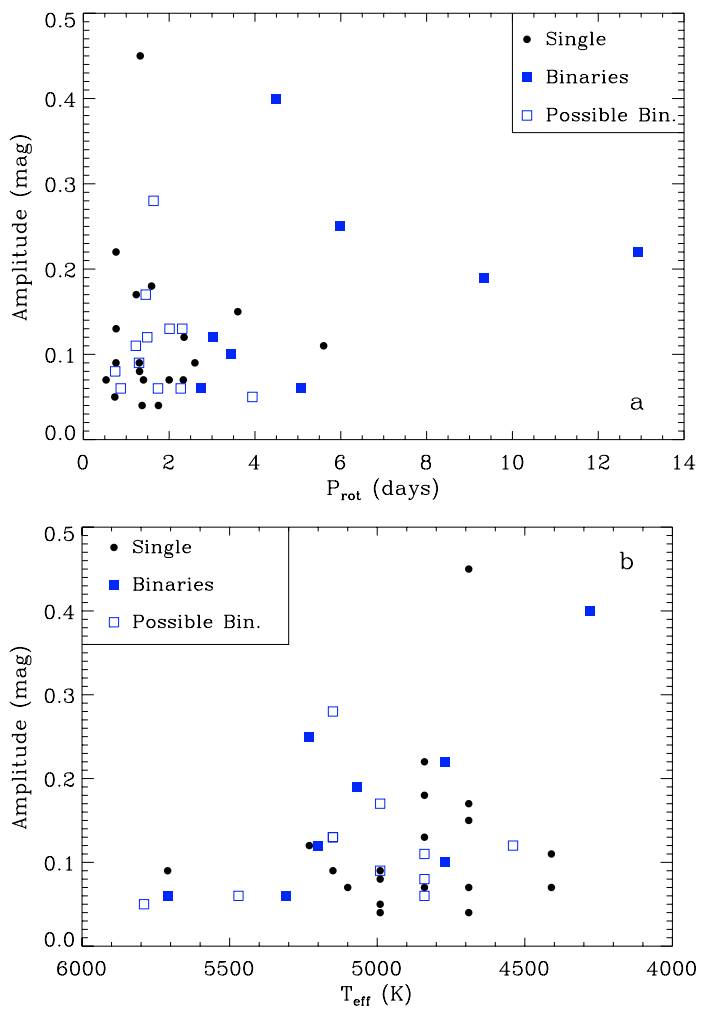

Fig. 9. $V$ light-curve amplitude for the sources in our sample plotted against $P_{\text {rot }}$ a) and $T_{\text {eff }} \mathbf{b}$ ).

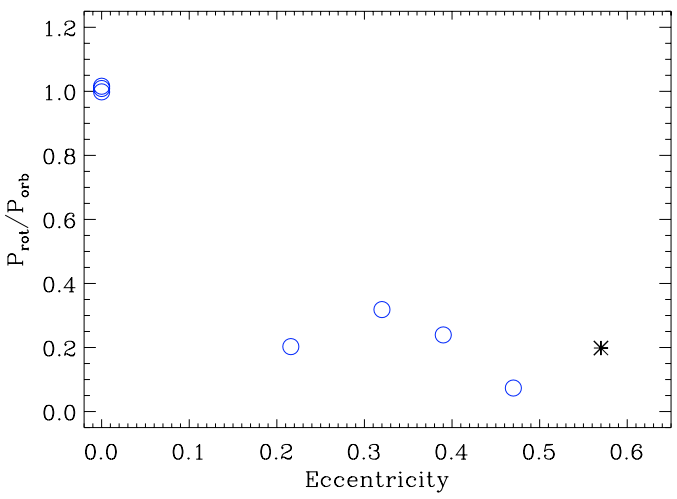

Fig. 10. $P_{\text {rot }} / P_{\text {orb }}$ versus eccentricity for the spectroscopic binaries with solved orbits (open circles). The position of the PMS eclipsing binary system KH 15D, belonging to NGC 2264 open cluster, is marked by an asterisk.

values well below the cut-off period of 7.56 days found by Melo et al. (2001) for orbital circularization in PMS binaries. The only system with a circular orbit and a $P_{\text {orb }}>7.56$ is 1RXS J035030.9-135527, but, due to its kinematics and the low lithium content, it is most likely an evolved active binary of the RS CVn type (Covino et al. 2001).

The remaining four systems have rather high orbital eccentricities $0.22 \leq e \leq 0.47$. All these systems are nonsynchronous with a ratio $P_{\text {rot }} / P_{\text {orb }}$ lower than about 0.35 , as shown in Fig. 10. Also displayed in this diagram is the eccentric PMS eclipsing system KH 15D, a member of the very young open cluster NGC 2264 (Hamilton et al. 2005; Winn et al. 2006), which fits very well the trend shown by the binaries in our sample.
Table 4. Circularization and pseudo-synchronization times.

\begin{tabular}{|c|c|c|c|c|c|}
\hline 1RXS J & $e$ & $\tau_{\text {circ }}$ & $\tau_{\text {sync }}$ & $\tau_{\text {pseudo }}$ & Age \\
\hline $052922.5+004112$ & 0 & 10 & 0.2 & 1 & 10 \\
\hline $053043.1-043453$ & 0.32 & $2 \times 10^{4}$ & 140 & 70 & 1 \\
\hline $053202.1-073153$ & 0.47 & $1 \times 10^{7}$ & $1 \times 10^{5}$ & $1 \times 10^{4}$ & 5 \\
\hline $054124.0-032446$ & 0 & 5 & 0.1 & 0.5 & 5 \\
\hline
\end{tabular}

As pointed out by Hut (1981), in a binary with an eccentric orbit the tidal interaction between the binary components is stronger around periastron, so that the equilibrium state is reached at a value of $P_{\text {rot }}$ which is less than $P_{\text {orb }}$ (pseudo-synchronization) and their ratio depends only on the orbital eccentricity. Using Hut's Eq. (42), one finds that the pseudo-synchronization periods are $10.58,24 \mathrm{~d} .85,18.30$, and 9d43, for 1RXS J044059.2-084005, 1RXS J053043.1043453, 1RXS J053202.1-073153 and RX J0539.8-0205, respectively. All these periods are significantly longer than the photometric (rotation) periods. This could indicate that these stars have not yet reached the pseudo-synchronization equilibrium. For KH 15D, a pseudo-synchronization period of 13.2 days is found with the value of $P_{\mathrm{orb}}=48.38$ and $e \simeq 0.57$ (Hamilton et al. 2005; Winn et al. 2006). These authors report a rotation period of 9.6 days, indicating that this binary has not reached the pseudo-synchronization condition yet, consistently with the scenario holding for the eccentric binaries in our sample. Moreover, Herbst \& Moran (2005) point out that KH 15D is also a weak X-ray emitter for its age and mass and argue that this could be related with its high eccentricity which brings periodically the two components close together affecting their magnetospheres. The binaries in our sample do not present any anomalous level of X-ray luminosity. However, we have no system with such a high eccentricity.

With the aim of further investigating this systematic difference, we evaluated the time scales for pseudo-synchronization, $\tau_{\text {pseudo }}$, following the guidelines of Hut (1981), for the systems in which the radius, mass, and luminosity are known for both components, i.e. the four systems listed at the bottom of Table 3. These time scales, together with circularization and synchronization time scales from Zahn (1989), $\tau_{\text {circ }}$ and $\tau_{\text {sync }}$, respectively, are listed in Table 4.

The circularization times for the two systems with $e \simeq 0$ are of the same order of the system age and the synchronization times are considerably shorter, in good agreement with the observations that indicate synchronous systems. For the two highly eccentric systems, $\tau_{\text {circ }}$ is instead several orders of magnitude larger than the age, implying that these binaries did not yet have the time to attain circularization and very likely will never circularize their orbits, at least during their main-sequence life. Moreover, for these latter systems, the time scale for pseudosynchronization is much greater than their age, in agreement with the observation of rotation periods shorter than the pseudosynchronization periods predicted by Hut's theory. This implies that the spin-up of the stars contracting along their evolutionary tracks occurs on a time scale much shorter than $\tau_{\text {pseudo, }}$, at least for 1RXS J053202.1-073153, and is then the dominating effect. However, for 1RXS J053043.1-043453, the value of $\tau_{\text {pseudo }} \approx 70 \mathrm{Myr}$ is comparable with the time-scale of the spinup process lasting from the PMS to the ZAMS phase, so that the two competitive effects of spin-up and slow-down are at work, giving rise to the observed rotational period of about 13 days. 
For a small sample of eight chromospherically active binaries, Hall (1986) found instead that, in five cases, the rotation periods are very close to those predicted by the pseudosynchronization theory. However, they are all binaries of the RS CVn or BY Dra type, hence much older than the systems investigated in the present paper.

\section{Discussion}

Let us discuss in more detail the rotation period distribution we find for our sparse population of Orion PMS stars.

In the WTTS regime, in which most of our targets fall, the PMS stars display a very low or negligible infall activity and accretion-disk evidence. Our sample of sparse WTTS has a flatter distribution of periods with respect to that of the ONC. In addition, the median value of about 1.5 days is much shorter than that of ONC stars in the same mass bin, also including the contribution of the spectroscopic binary components.

The period distribution found by Herbst et al. (2002) for ONC stars with $M \geq 0.25 M_{\odot}$ is bimodal with peaks near to 2 and 8 days. For low-mass stars (below $\approx 0.25 M_{\odot}$ ) the distribution is instead unimodal and peaked at a period of about 2 days. In the mass range of the stars investigated by us $\left(M \geq 0.7 M_{\odot}\right)$, the period distribution for the ONC stars becomes unimodal with a peak at about 7 days and a tail towards shorter periods. This behavior could be explained in terms of the effectiveness and duration of the disk-locking mechanism which is dependent on the star mass and environmental conditions. Hartmann (2002) proposed a relationship that gives a lower limit to the braking timescale as a function of the stellar mass, the mass accretion rate, and the ratio of the actual rotation rate to the breakup velocity. For the ONC stars of $0.3 M_{\odot}$, Hartmann (2002) deduced a braking time-scale significantly longer than the ONC age (1 Myr), i.e. the disk-locking may be ineffective to slow-down ONC lowmass stars, as it is observed. The same relationship applied to solar-mass stars, for which the accretion rate is greater at least by one order of magnitude, gives values of the braking time-scale of the order of $0.5 \mathrm{Myr}$ (Rebull et al. 2004), i.e. the disk-locking is more effective and this justifies the trend we find from the Herbst et al. (2002) data, with the peak at longer periods and the tail at shorter ones. However, different accretion rates in stars of similar mass and age, as well as the topology of the stellar magnetic field and its interaction with the disk material could greatly affect the disk-locking time-scales accounting for the observed range of rotational periods. Moreover, from 1 to $2 \mathrm{Myr}$ the evolution of the rotation-period distribution depends strongly on how long the disks last and on all the factors that can affect this duration, like the star mass and environmental conditions (see, e.g., Lamm et al. 2005; Nordhagen et al. 2006). The main factor appears to be the stellar mass. For higher mass stars $\left(M \geq 0.3 M_{\odot}\right)$, disk-locking seems to be more frequent and long-lasting than for lower-mass stars. However, there is growing evidence that environmental factors play a role in establishing the rotational properties of the members of a cluster. If disks last for long time (probably because they are in a mild environment lacking an $\mathrm{O}$ star) then a larger fraction of the stars does not spin up with time. If the disks last a shorter time, like it is believed to occur in NGC 2264 where O stars are present, then more stars could lose their disks sooner and start to spin up. Indeed, the long-period peak of the distribution for NGC 2264 is found at about 4 days (Lamm et al. 2005).

In this scenario, the period distribution of the Orion WTTS population represented by our sample can be interpreted as the natural evolution of the angular momentum of solar-mass stars at ages typically greater than $1 \mathrm{Myr}$ and with an age spread of about 10 Myr.

At this evolutionary stage Wolff et al. (2004) find that, for solar or greater stellar mass, the transition from convective to radiative tracks could favor the decoupling of the angular momentum at the surface of the stars from the angular momentum in the interior. In this case rotation rates vary inversely with the stellar radius (Rebull et al. 2004) and this could account for the WTTS distribution of periods found here. So, the age spread around a critical evolutionary phase is, in our opinion, the main responsible for the observed period distribution of the sparse population of young stars investigated by us.

The subsequent evolution along the radiative tracks or near the ZAMS is characterized by the conservation of the angular momentum. After the stars reach the main sequence, an additional spin down by magnetic winds takes place as shown by the comparison of the two bimodal distributions of $\alpha$ Per and Pleiades, displaced each other by an amount that can be ascribed to the action of the magnetic braking mechanism operating at the different ages of the two clusters.

In spite of the limited star sample, it appears that the components of binary systems tend to show a different rotation distribution, with a greater incidence of slow rotators, as expected from the tidal interaction between orbit and rotation. Thus, for each cluster, photometric and spectroscopic data would be needed in order to state whether or not a star is a spectroscopic binary with a sufficiently short orbital period such that the rotation of the components could be significantly affected by spin-orbit angular momentum exchange due to tidal interaction.

\section{Conclusions}

We presented new determinations of rotation periods for a sample of 39 young stars in the Orion complex by means of a time series analysis of a large amount of photometric data collected by us over more than seven years. These stars were discovered as optical counterparts of X-ray sources in the RASS by Alcalá et al. (2000a) and Frasca et al. (2003) using both low- and highresolution spectroscopy.

From their position on the HR diagram and the comparison with PMS evolutionary tracks and isochrones, we have inferred masses in the range $0.7-2.5 M_{\odot}$.

The rotation periods display a flat single-peaked distribution centered at a value of about 1.5 days, significantly shorter than the peak found for stars belonging to the ONC and in the same mass range. This behavior is consistent with the standard angular momentum evolution scenario, considering that the sparse population investigated by us is composed of stars typically older than the ONC ones (1 Myr) and with an age spread of several Myr. The stars in our sample, nearly all WTTS, are in an evolutionary phase in which the disk-locking mechanism is no longer effective and any eventual accretion occurs at a very low rate, so that a spin-up takes place, due to the decrease of the moment of inertia produced by the star's contraction and eventually to the transition from convective to radiative PMS evolution, followed by a redistribution of the angular momentum inside the star.

We also found that the components of close binary systems tend to rotate more slowly than single stars, due to the tidal coupling between orbital and rotational motion. In particular, all the systems with circular orbits are also synchronized, consistently with the circularization and synchronization time-scales, while the systems with eccentric orbits have components that are not tidally locked. For the latter we evaluated, according to the relationships proposed by Hut (1981), the pseudo-synchronization 
periods at periastron, that come out to be always longer than the observed ones. The evaluation of pseudo-synchronization timescales indicates that the tidal coupling only acts as an additional braking mechanism during their spin-up phase.

To confirm the trend we found for the WTTS period distribution, it is advisable to observe larger star samples with a very high photometric precision and long enough time baselines. The latter would enable us to pick up the stars with longer periods and with small-amplitude variations.

In any case, these results show the strong capability of observations of stellar rotation as probes of fundamental processes that occur during the formation and early evolution of stars and binary systems.

Acknowledgements. We thank the technical staff of the OAC, P. Bruno, G. Carbonaro, A. Distefano, M. Miraglia, A. Miccichè, and G. Occhipinti, for the valuable support with the observations. We are grateful to the anonymous referee for useful comments and suggestions that helped to improve the manuscript. This research has been supported by INAF and Italian MIUR. M.F. was supported by the Spanish grants AYA2004-05395 and AYA2004-21521-E.

\section{References}

Alcalá, J. M., Terranegra, L. Wichmann, et al. 1996, A\&AS, 119, 7

Alcalá, J. M., Krautter, J., Covino, E., et al. 1997, A\&A, 319, 184

Alcalá, J. M., Covino, E., Torres, G., et al. 2000a, A\&A, 353, 186

Alcalá, J. M., Covino, E., Sterzik, M. F., et al. 2000b, A\&A, 355, 629

Bouvier, J., Wichmann, R., Grankin, K., et al. 1997, A\&A, 318, 495

Broeg, Ch., Fernández, M., \& Neuhäuser, R. 2005, Astron. Nachr., 326, 134

Chavarría-K, C., Terranegra, L., Moreno-Corral, M. A., \& de Lara, E. 2000, A\&AS, 145, 187

Cohen, R. E., Herbst, W., \& Williams, E. C., 2004, AJ, 127, 1602

Covino, E., Catalano, S., Frasca, A., et al. 2000, A\&A, 361, L49

Covino, E., Melo, C., Alcalá, J. M., et al. 2001, A\&A, 375, 130

Covino, E., Frasca, A., Alcalá, J. M., et al. 2004, A\&A, 427, 637

Cutri, R. M., Skrutskie, M. F., Van Dyk, S., et al. 2003, Explanatory Supplement to the 2MASS All Sky Data Release

D’Antona, F., \& Mazzitelli, I. 1997, Mem. Soc. Astron. Ital., 68, 807

Frasca, A., Alcalá, J. M., Covino, E., et al. 2003, A\&A, 405, 149
Freire Ferrero, R., Frasca, A., Marilli, E., \& Catalano, S. 2004, A\&A, 413, 657 de Jager, C., \& Nieuwenhuijzen, H. 1987, A\&A 177, 217

de Zeeuw, P. T., Hoogerwerf, R., de Bruune, J. H. J., Brown, A. G. A., \& Blaauw, A. $1999, \mathrm{AJ}, 117,354$

Genzel, R., \& Stutzki, J. 1989, ARA\&A, 27, 41

Greve, A., Castles, J., \& McKeith, C. D. 1994, A\&A, 284, 919

Hall, D. S. 1986, AJ, 309, L83

Hamilton, C. M., Herbst, W. J., Vrba, F. J., et al. 2005, AJ, 130, 1896

Hartmann, L. 2002, ApJ, 566, L29

Hauschildt, P. H., Allard, F., \& Baron, E. 1999, ApJ, 512, 377

Herbst, W., \& Moran, E. C. 2005, ApJ, 630, 400

Herbst, W., \& Mundt, R. 2005, ApJ, 633, 967

Herbst, W., Bailer-Jones, C. A. L., Mundt, R., et al. 2002, A\&A, 396, 513

Hog, E., Fabricius, C., \& Makarov, V. V., et al. 2000, A\&A, 355, L27

Horne, J. H., \& Baliunas, S. L., 1986, ApJ, 302, 757

Hut, P. 1981, A\&A, 99, 126

Kukarkin, B. V., Kholopov, P. N., Pskovsky, Y. P., et al. 1971, General Catalogue of Variable Stars, 3rd edn.

Landolt, A.U. 1992, AJ, 104, 340

Lamm, M. H., Mundt, R., Bailer-Jones, C. A. L., \& Herbst, W. 2005, A\&A, 430, 1005

Marilli, E., Catalano, S., \& Frasca, A. 1997, Mem. Soc. Astron. Ital., 68, 895

Melo, C. H. F., Covino, E., Alcalá, J. M., \& Torres, G. 2001, A\&A, 378, 898

Messina, S., Pizzolato, N., Guinan, E. F., \& Rodonò, M. 2003, A\&A, 410, 671

Neuhäuser, R., Sterzik, M. F., Schmitt, J. H. M. M., Wichmann, R., \& Krautter, J. 1995, A\&A, 297, 391

Nordhagen, S., Herbst, W., Rhode, K. L., \& Williams, E. C. 2006, AJ, 132, 1555

Palla, F., \& Stahler, S. W. 1999, ApJ, 525, 772

Parenago, P. P. 1954, Trudy Gosud. Astron. Inst. Shternberga, 25, 1

Pizzolato, N., Maggio, A., Micela, G., Sciortino, S., \& Ventura, P. 2003, A\&A, 397, 147

Randich, S., 1998, ASP Conf. Ser., 154, 501

Rebull, L. M., Wolff, S. C., \& Strom, S. E. 2004, AJ, 127, 1029

Rebull, L. M., Stauffer, J. R., Megeath, S. T., Hora, J. L., \& Hartmann, L. 2006, ApJ, 646, 297

Roberts, D. H., Lehar, J., \& Dreher, J. W., 1987, AJ, 93, 968

Rosino, L., \& Cian, A. 1961, Mem. Soc. Astron. Ital., 32, 297

Scargle, J. D. 1982, ApJ, 263, 835

Winn, J. N., Hamilton, C. M., Herbst, W. J., et al. 2006, ApJ, 644, 510

Wolff, S. C., Strom, S. E., \& Hillenbrand, L. A. 2004, ApJ, 601, 979

Zahn, J.-P. 1989, A\&A, 220, 112

Zahn, J.-P., \& Bouchet, L. 1989, A\&A, 223, 112 
E. Marilli et al.: Rotational periods of WTTS in Orion, Online Material $p 1$

\section{Online Material}




\section{Appendix A: Individual objects}

A dedicated analysis of the individual objects in our sample shows a wide variety of photometric behaviors that appear to be related both to their different evolutionary status and to their nature of single or multiple systems.

\section{A.1. 1RXS J035030.9-135527}

1RXS J035030.9-135527 is a spectroscopic binary whose orbital and physical parameters were reported by Covino et al. (2001). Its sky location and kinematics seems to indicate that we are seeing an evolved system with characteristics typical of the RS CVn binaries. The relatively low lithium content, the circular orbit and the nearly synchronous rotation, in spite of the rather long orbital period, give support to this scenario. For this reason, we have preferred to exclude this system from the rotationperiod distribution.

\section{A.2. $1 R X S$ J035637.8-132719}

This object, which is projected on the sky close to the previous one, also appears located rather far away from the bulk of the Orion complex. However, its radial velocity, $+17 \pm 2 \mathrm{~km} \mathrm{~s}^{-1}$, determined from three unpublished FEROS spectra, appears consistent with that of the sparse Orion WTTS population (Alcalá et al. 2000a). Its proper motion, $\mu_{\alpha} \cos \delta=-11.4 \mathrm{mas} \mathrm{yr}^{-1}, \mu_{\delta}=$ $-10.5 \mathrm{mas} \mathrm{yr}^{-1}$, from TYCHO2 (Hog et al. 2000) is also consistent with those of the WTTS in Orion (Frasca et al. 2003). The $\mathrm{K} 1 \mathrm{~V}$ spectral type and the $v \sin i$ value of about $80 \mathrm{~km} \mathrm{~s}^{-1}$ have been determined for the first time in this work by applying the ROTFIT code developed by us (Frasca et al. 2003; Freire Ferrero et al. 2004) to the aforementioned FEROS spectra. The relatively high lithium abundance indicates a very young star in the PMS phase that, in spite of its outlying location, might be connected with the Orion complex. A possible period of 1.37 days is suggested by the OAC photometry.

\section{A.3. $1 R X S$ J044059.2-084005}

Hierarchical triple system. The inner spectroscopic binary has a systemic velocity consistent with the Orion SFR, but it has been classified as a main-sequence binary with components already onto the ZAMS by Covino et al. (2001) on the basis of the Li I abundance. A photometric period $P_{\text {rot }} \simeq 2.7$ days, much shorter than the orbital one, is indicated by our OAC photometry.

\section{A.4. 1RXS J050040.9-045617}

Possible binary system, according to the variable RV and CCF width (Frasca et al. 2003). The period derived from the OAC data ( $P_{\text {rot }}=2.27$ days) is consistent with the $v \sin i$.

\section{A.5. 1RXS J050105.6+064209}

Single star with an indication of a very short photometric period from the OAC data (0.53 days). This uncertain period is, however, rather inconsistent with the fairly low $v \sin i$, resulting in an inclination of the rotation axis of only $5^{\circ}$, so that it could be a spurious one. A more reliable period of 2.33 days results from the analysis of the few SPM 2003 data.

\section{A.6. 1RXS J050306.4+054851}

Possible binary system, based on the RV variation detected by Frasca et al. (2003). The relatively long photometric period (3.94 days) is not consistent with the $v \sin i=80 \mathrm{~km} \mathrm{~s}^{-1}$ measured by means of medium-resolution spectra by Frasca et al. (2003), yielding a $\sin i$ factor of 1.6. However, this $v \sin i$ value could be a result of blended binary components. A $v \sin i=45 \mathrm{~km} \mathrm{~s}^{-1}$ for each component would reconcile the inconsistency.

\section{A.7. $R X$ J0503.8-1130}

An uncertain period of about 1.75 days with a very small LC amplitude (only 0 . 04 ) is deduced from the SPM photometry obtained in 1999. However, this period is fully consistent with the $v \sin i$.

\section{A.8. $R X$ J0507.8-0931}

The CCF shape suggests a possible double-lined SB (Alcalá et al. 2000a). The rotation period of about 1.2 days, determined both from the CA and the SPM data, is fully consistent with the $v \sin i$. The LC obtained at CA displays a double wave indicative of two distinct active longitudes in that observing season.

\section{A.9. $R X$ J0509.0-0315}

Asymmetric CCF. A relatively short period (0.734 days) is deduced from the LC obtained in 1999 at SPM. The wave amplitude is, however, small $(0 \mathrm{~m} 05)$ and the period is only marginally consistent with the $v \sin i$.

\section{A.10. RX J0511.7-0348}

A rotation modulation with a period of about 1.3 days was detected in the SPM data obtained in 1999. No modulation was visible 4 years later.

\section{A.11. 1RXS J051754.9-070819}

Star falling just above the upper envelope of young open clusters in the $W(\mathrm{Li})-T_{\text {eff }}$ diagram (Alcalá et al. 2000a). The short photometric period (0.76 days) appears inconsistent with the $v \sin i$ $\left(18 \mathrm{~km} \mathrm{~s}^{-1}\right)$, resulting in an inclination of the rotation axis of only $4^{\circ}$.

\section{A.12. $R X J 0518.0+0712$}

Suspected spectroscopic multiple system with an asymmetric CCF (Alcalá et al. 2000a). No periodic modulation was detected in the OAC data. A fairly short period (0.872 days), in agreement with the relatively high $v \sin i$, was instead detected from the SPM photometry taken in 1999. Strong lithium.

\section{A.13. $R X J 0518.6+0959$}

Single PMS star. We found two different periods from the OAC (2.71: days) and the SPM (1.59 days) data sets taken in different epochs. Both periods are consistent with the $v \sin i$ and the stellar radius, but the SPM light-curve is cleaner, i.e. it has a higher amplitude and the period is more firmly established. 


\section{A.14. 1RXS J052254.9+085803}

SB2 binary (G. Torres, private communication). The photometric period is nearly equal to the orbital one (synchronous system). The relatively low lithium content could be indicative of a couple of stars in an evolutionary stage later than the PMS phase. The Li I $\lambda 6708 \AA$ line is weaker than the nearby Ca I line $\lambda 6718 \AA$.

\section{A.15. 1RXS J052345.0-075343}

The Li I $\lambda 6708$ line is not detected by Frasca et al. (2003) from medium-resolution spectra. However, its RV and kinematics are fully consistent with the Orion SFR. The rotation period $P_{\text {rot }}=$ 1.4 days is marginally consistent with the $v \sin i$ estimate given by Frasca et al. (2003) by means of medium-resolution spectra.

\section{A.16. 1RXS J052423.1-064007}

Confirmed as a WTTS by Frasca et al. (2003) from both its high LiI EW and kinematics, it is also the WTTS with the shorter photometric period in our sample ( $P_{\text {rot }}=0.53$ days). This period is marginally consistent with the $v \sin i$ estimate given by Frasca et al. (2003) by means of medium-resolution spectra. Further follow-up high-resolution spectroscopic observations are mandatory.

\section{A.17. RX J0528.0-0053}

Well defined photometric period of 2.305 days from the CA data. The SPM photometry taken in 1999 confirms this period. Unfortunately the $v \sin i$ has not been measured yet. According to its position on the sky, the object is probably associated with the part of the Orion complex closest to the Sun, Ori OB1A, for which a distance of $336 \mathrm{pc}$ has been evaluated by de Zeeuw et al. (1999). Lithium is strong and the CCF suggests a possible blended SB2.

\section{A.18. 1RXS J052847.3+004840}

Well defined photometric period of 1.636 days from the OAC data. Unfortunately the $v \sin i$ has not been measured yet. Also this source is spatially consistent with Ori OB1A. Strong lithium and suspected SB2 on the basis of the CCF shape.

\section{A.19. 1RXS J052922.5+004112}

Eclipsing spectroscopic binary. It is indeed a hierarchical triple, whose components have been well studied and characterized by (Covino et al. 2004) also based on the photometric data taken at $\mathrm{OAC}$ and CA and displayed in Fig. 1. The analysis of the out-ofeclipse data indicates the presence of rotational modulation due to photospheric spots.

\section{A.20. 1RXS J053005.6+004121}

Classified as G2 by Alcalá et al. (2000a) and G3 by Frasca et al. (2003), this is the brightest object in the sample. It coincides spatially with Ori OB1A, for which a distance of $336 \mathrm{pc}$ was assumed. The photometric period of 1.304 days is clearly detected in the OAC data and is consistent with the $v \sin i$. Strong lithium and suspected SB2 on the basis of the CCF shape.

\section{A.21. 1RXS J053043.1-043453}

SB2 system with two nearly equal K2-3 type components discovered by Covino et al. (2001). The photometric period of about 13 days is roughly $1 / 3$ of the orbital one and this is consistent with the $v \sin i$ 's and radii quoted by Covino et al. (2001) that confirm the non-synchronous rotation. This period is found in two OAC data sets obtained in different seasons and also in the CA data combined with the OAC ones (see Fig. 2); the time span of only 9 days for the CA observations prevents the detection of such a long period.

We notice that for a system with the orbital period and eccentricity of 1RXSJ053043.1-043453, the rotation period expected in case of pseudo-synchronization at periastron is nearly 25 days (Hut 1981), that is about twice the period derived from the photometry, in agreement with the expectations from pseudosynchronization time scales. However, because of this coincidence, we may not completely rule out the possibility that the rotation of the components be synchronized with the orbital velocity at periastron and that the period derived from the photometry actually corresponds to half of the rotation period.

\section{A.22. $1 R X S$ J053055.8+101506}

The photometric modulation is more evident in the SPM data with a $\Delta R$ amplitude of about $0.13 \mathrm{mag}$. However, the periods found in the SPM and OAC data sets are slightly different. Suspected multiple SB.

\section{A.23. $1 R X S$ J053202.1-073153}

SB2 system with the longer orbital period in our sample $\left(P_{\text {orb }} \simeq\right.$ 47 days) with nearly equal K2-3 type components (Covino et al. 2001). The photometric period, detected in the CA data at a confidence level of about $97 \%$, is 3.44 days, which is fairly consistent with the $v \sin i$ values of the system components (20 and $22 \mathrm{~km} \mathrm{~s}^{-1}$ for the primary and secondary component, respectively), giving rise to a $\sin i$ factor of about 0.8 . With this value, the dynamical masses $M_{1,2} \sin ^{3} i$, measured by Covino et al. (2001), lead to absolute masses of about $2-2.3 M_{\odot}$ in marginal agreement with those inferred from the evolutionary tracks $\left(M_{1,2} \approx 1.4 M_{\odot}\right)$. Based on the OAC data we are not able to clearly detect any periodicity, while the SPM photometry taken in 1999 displays a modulation with a period of about 4 days, i.e. near to that detected at CA. The pseudosynchronization period for this system would be about 18.5 days, however, from both the $v \sin i$ values and the period derived from the photometry, the asynchronism between orbital and rotational motion appears a real one.

\section{A.24. $R X$ J0532.4+0131}

Visual binary with a separation of about $9^{\prime \prime}$ composed of two PMS stars. A possible photometric period of nearly exactly two days has been detected for the brighter component with the SPM data acquired both in 1999 and 2003. The fainter star displays a clear modulation with a period of 5.6 days.

\section{A.25. $1 R X S$ J053231.8-042135}

Alcalá et al. (2000a) report this stars as a close $\left(1^{\prime \prime}\right)$ visual pair and list two very different values of $v \sin i\left(21\right.$ and $\left.35 \mathrm{~km} \mathrm{~s}^{-1}\right)$. The very young age we found from its position on the HR diagram could be affected by the contribution of the two unresolved 


\section{E. Marilli et al.: Rotational periods of WTTS in Orion, Online Material p 4}

visual components to the observed flux. We find a well defined photometric wave with a period of 1.23 days that is marginally consistent with both $v \sin i$ values. However, accounting for the duplicity, the disagreement is reduced.

\section{A.26. 1RXS J053236.6-052254}

Already known as a variable (HR Ori) from the early work of Parenago (1954). Rosino \& Cian (1961) report it as a variable with a small amplitude and a photographic magnitude in the range $m_{\mathrm{pg}}=13.2-13.8 \mathrm{mag}$, but no indication of periodicity. It is the PMS star of our sample with the largest amplitude of the rotational modulation (nearly half magnitude). The photometric period of about 1.33 days was found both in the OAC and SPM data sets.

\section{A.27. 1RXS J053304.9-075850}

Small-amplitude variations giving rise to uncertain values of the photometric period. The value of $P_{\text {rot }} \simeq 11$ days found from the OAC data set is inconsistent with the star radius and $v \sin i$ ( $\sin i=3.5$ ), while the period of about 2.6 data found from the SPM photometry is consistent with these data $(\sin i \simeq 0.83)$.

\section{A.28. RXS J0533.1+0224}

Suspected spectroscopic multiple with an asymmetric CCF (Alcalá et al. 2000a). Observed only at SPM. A possible period of about 1.49 days is found. Strong lithium.

\section{A.29. $R X J 0534.7+1114$}

Star falling just above the upper envelope of young open clusters in the Li I- $T_{\text {eff }}$ diagram (Alcalá et al. 2000a). Observed only at SPM. No rotational modulation has been detected.

\section{A.30. $R X$ J0535.0-0411}

The object shows strong lithium and is suspected to be a doublelined SB (Alcalá et al. 2000a) on the basis of its CCF shape. A clear rotational modulation with a period of 2.35 days has been detected only from the OAC photometry. No rotational modulation has been detected from the SPM data.

\section{A.31. 1RXS J053534.2-015227}

Given by Alcalá et al. (2000a) as a possible binary on the basis of double blended peaks in the cross correlation function. Small amplitude of the photometric wave ( 0 . 06$)$.

\section{A.32. $R X 0536.7+0907$}

SPM data taken in 2000 show a possible modulation with a period of about 0.77 days. Subsequent observations performed at OAC in 2005-2006 display instead a clear variation $(\Delta V \simeq 0.22)$ with a period of 0.762 days, substantially confirming the early detection by SPM data. However, the $\sin i$ value deduced is fairly low.

\section{A.33. $R \times 0539.3+0918$}

Given by Alcalá et al. (2000a) as a possible binary on the basis of the variable $R V$ and a broad, asymmetric CCF. G. Torres (private communication) reports also a variable $R V$ and classify it as a possible SB2 system, suggesting that the $v \sin i$ of the two components is nearly the same and roughly one half than the value of $108 \mathrm{~km} \mathrm{~s}^{-1}$ reported by Alcalá et al. (2000a). The rotation period of about 1.30 days detected both from the OAC and SPM photometry is well consistent with both $v \sin i$ determinations.

\section{A.34. RX J0539.8-0205}

Quoted by Alcalá et al. (2000a) as a possible spectroscopic multiple on the basis of the CCF shape. Confirmed as a SB2 system by $\mathrm{G}$. Torres (private communication), which also solved the orbit and kindly provided us with the orbital parameters. We detected, with a fairly good confidence level, a rotation period of about 4.5 days on the basis of our longer data set taken at OAC from December 1999 to February 2000 and a large wave amplitude $(\Delta V \simeq 0.4)$. Though with very few points, in another data set obtained in 2006 at OAC we confirm such a large amplitude with a variation time-scale compatible with the 4.5 -days period.

This object is another example of a binary with eccentric orbit for which we find a photometric period shorter than the theoretical period of pseudo-synchronism at periastron (9.4 days). However, also in this case, the observed period is roughly one half of the predicted one. Unfortunately, no $v \sin i$ value could be determined and thus we cannot check the actual synchronization degree of the components.

\section{A.35. $1 R X S$ J053956.8+095638}

Quoted by Alcalá et al. (2000a) as a SB1 system on the basis of variable $R V$. The $R V$ variation on a very long time-scale has been confirmed by $\mathrm{G}$. Torres (private communication) who suggests a SB1 system with a period longer than 1 year. As such, we have treated this object as a single star. We determined a short rotation period (0.764 days).

\section{A.36. $1 R X S$ J054014.0-070832}

Suspected double-lined SB (Alcalá et al. 2000a) on the basis of its CCF shape. Differential $V$ and $R$ photometry has been gathered only at the CA Observatory. A possible rotation period of about 2.0 days has been found from these data. This value is compatible with the $v \sin i$ value of $36 \mathrm{~km} \mathrm{~s}^{-1}$.

\section{A.37. 1RXS J054033.2-012157}

Possible SB2 with partially blended components (Alcalá et al. 2000a). A well defined modulation with a period of 1.454 days has been found both from the OAC and CA data taken in different seasons. Unfortunately, no $v \sin i$ value could be determined and thus we could not evaluate the inclination of the rotation axis.

\section{A.38. 1RXS J054124.0-032446}

Double-lined spectroscopic binary with orbital period of almost exactly five days. The photometric period detected from the CA data is practically identical to the orbital one. The highest peak of the cleaned periodogram of the OAC data corresponds to a period of about 1.25 days, which is exactly $1 / 4$ of the orbital period and is very likely an overtone of the main period. By merging the $\mathrm{CA}$ and OAC data sets, obtained one month apart, this 


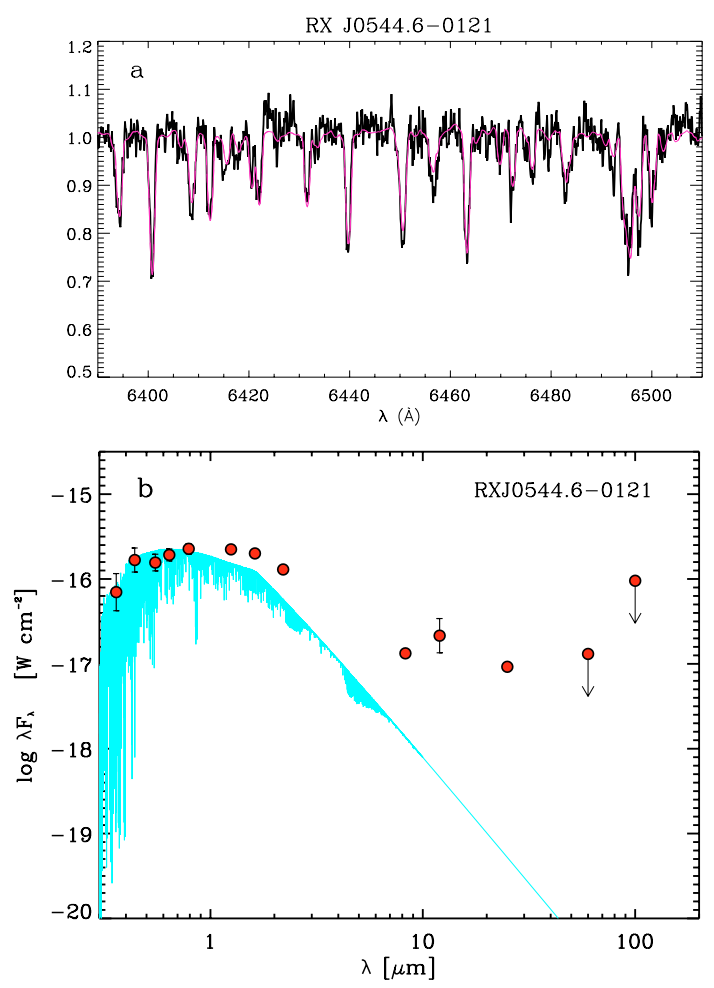

Fig. A.1. a) Observed FOCES spectrum of RX J0544.6-0121 at red wavelengths (thick black line) with the $\mathrm{K} 1 \mathrm{~V}$ spectral template broadened at $v \sin i=18 \mathrm{~km} \mathrm{~s}^{-1}$ over-plotted (thin red line). b) Observed SED of RX J0544.6-0121 built up with optical and IR fluxes (dots) together with the NextGen spectrum at the star temperature $\left(T_{\text {eff }}=5000 \mathrm{~K}\right)$ matched to the optical part of SED. The strong IR excess is apparent.

short period is no longer detectable and a periodicity of about 5.76 days comes out. This indicates that the true rotation period must be about 5 days and that the system is very likely synchronous. From the radii, $v \sin i$ 's and the rotational/orbital pe$\operatorname{riod}\left(P_{\text {phot }} \simeq 5\right.$ days), we derive a $\sin i$ factor of about 0.88 that, combined with the dynamical masses, $M_{1} \sin ^{3} i=1.307 M_{\odot}$, $M_{2} \sin ^{3} i=0.881 M_{\odot}$, obtained by Covino et al. (2001), yields mass values of 1.9 and $1.3 M_{\odot}$ for the primary and secondary component, respectively. These values are in very good agreement with those derived from the evolutionary tracks by Palla \& Stahler (1999).

\section{A.39. $R X J 0544.6-0121$}

Reported as a variable star (V523 Ori) in the GCVS (Kukarkin et al. 1971). This object displays the most peculiar extinction, $A_{V}=3$. 0 , and is clearly surrounded by a reflection nebula. It could be a very reddened star.

Due to these peculiarities, we have checked carefully its characteristics. The spectral type (K1 V) and $v \sin i\left(18 \mathrm{~km} \mathrm{~s}^{-1}\right)$ have been re-determined in this work by applying the RoTFIT code developed by us (Frasca et al. 2003; Freire Ferrero et al. 2004) to a FOCES high-resolution spectrum (Fig. A.1a). From the analysis of a low-resolution B\&C spectrum (Alcalá et al. 1996) we deduced the same spectral type and an extinction $A_{V} \simeq 3$. 2 .

This star is also an IRAS infrared source. The spectral energy distribution, constructed with all the optical and infrared fluxes, displays a strong IR excess (see Fig. A.1b) indicative of abundant circumstellar matter around this object which could still be in a very early evolutionary stage, as also suggested by its position in the HR diagram.

Alcalá et al. (2000a) classify this object as a single star with a strong lithium. An uncertain period of about 3.6 days is detected from the SPM photometry acquired in 2000 . No modulation was seen in the subsequent run in 2003. 